\title{
Electroweak Reactions in the Non-Perturbative Regime of QCD
}

\author{
Ulf-G. Meißner
}

University of Berne, Institute for Theoretical Physics, CH-3012 Berne, Switzerland

\section{Contents}

1 Introduction 1

2 Chiral Perturbation Theory with Nucleons 2

2.1 Chiral Symmetry in QCD 2

2.2 Chiral Perturbation Theory (Mesons) 3

2.3 Inclusion of Matter Fields 5

2.4 Threshold Pion Photo- and Electroproduction $\quad 7$

2.5 Nucleon Compton Scattering 9

3 The Quark Structure of the Nucleon 12

3.1 Currents, Sizes and Form Factors of the Nucleon 12

3.2 A Toy Model: Quark Distributions from Form Factors 14

$\begin{array}{lll}3.3 & \text { Electroweak Currents } & 15\end{array}$

3.4 Parity-Violating Electron Scattering $\quad 18$

3.5 Neutrino and Antineutrino Scattering off Nucleons and Nuclei 19

3.6 Down and Dirty: QED, QCD and Heavy Quark Corrections 21

4 Summary and Outlook 25

\section{Introduction}

These lectures are concerned with the structure of hadrons at low energies, where the strong coupling constant is large. Most of the hadronic world discussed here will be made up of the light $\mathrm{u}, \mathrm{d}$ and $\mathrm{s}$ quarks since these are the constituents of the low-lying hadrons. The best way to gain information about the strongly interacting particles is thus the use of well-understood probes, such as the photon or the massive weak gauge bosons. At very low energies, the dynamics of the strong interactions is governed by constraints from chiral symmetry. This leads to the use of effective field theory methods which in the present context is called baryon chiral perturbation theory. In the first part of these lectures, I will briefly outline the basic framework of this effective field theory and use photo-nucleon processes to discuss the strengths and limitations of it. The basic degrees of freedom are the pseudoscalar Goldstone

Lectures delivered at the XXXII. Internationale Universitätswochen für Kern- und Teilchenphysik, Schladming, Styria, Austria, February 24 - March 6, 1993. 
bosons chirally coupled to the matter fields like e.g. the nucleons. The very lowenergy face of the low-lying baryons is therefore of hadronic nature, essentially pointlike Dirac particles surrounded by a cloud of Goldstone bosons. The information about the underlying quark structure is only indirect since one translates e.g. quark masses into pseudoscalar meson masses. Nevertheless, certain aspects of the quark structure of the nucleon (or other baryons) can be extracted by making clever use of the well-known couplings of the quarks to the electroweak gauge bosons. For that, it is important to understand the notion of hadron form factors, electromagnetic or weak ones. These encode certain aspects of the quark structure of the nucleon. In addition, parity-violating electron scattering off nucleons or nuclei or neutrinonucleon scattering allows one to measure the weak form factors related to the neutral currents which complement the information gained from the electromagnetic and weak charged current form factors. This will be the theme of the second part of these lectures. However, since one is dealing with small effects like e.g. left-right asymmetries in polarized electron scattering off protons at low energies, one also has to worry about the effects of the heavy quarks $\mathrm{c}, \mathrm{b}$ and $\mathrm{t}$ and about radiative corrections, which are nominally suppressed by powers of $(\alpha / 4 \pi)$ but sometimes enhanced by large logarithms or by some judiciously large factors. I will give a short state of the art summary on these topics at the end of these lectures.

Naturally, I can only cover a small fraction of the many interesting phenomena related to low energy hadron physics. I have chosen to mostly talk about the nucleon since after all it makes up large chunks of the stable matter surrounding us and also is a good intermediary between the nuclear and the high energy physicists present at this school. Most of the methods presented here can easily be applied to other problems, and as it will become obvious at many places, we still have a long way to go to understand all the intriguing features of the nucleon in a systematic and controlled fashion. Whenever possible, I will avoid to talk about models, with the exception of some circumstances where they offer some qualitative insight into certain aspects of hadron structure.

\section{Chiral Perturbation Theory with Nucleons}

\subsection{Chiral Symmetry in QCD}

Consider the QCD Lagrangian for the three light flavors $\mathrm{u}, \mathrm{d}$ and $\mathrm{s}$,

$$
\mathcal{L}_{Q C D}=\bar{q}\left(i \gamma_{\mu} D^{\mu}-\mathcal{M}\right) q+\mathcal{L}_{\text {gluon }}
$$

with $q=(\mathrm{u}, \mathrm{d}, \mathrm{s})$ and I have suppressed all color indices. The quark fields can be chosen such that the quark mass matrix is diagonal,

$$
\mathcal{M}=\left(\begin{array}{lll}
m_{u} & & \\
& m_{d} & \\
& & m_{s}
\end{array}\right)
$$

The entries in (2.2) are the current quark masses. At the typical hadronic scale of $\Lambda \simeq 1 \mathrm{GeV}$, these can be considered small. This holds certainly for the $\mathrm{u}$ and $\mathrm{d}$ quarks, the s quark is somewhat heavier $(\simeq 175 \mathrm{MeV})$ which makes it more difficult 
to deal with. To a good first approximation, it is, however, legitimate to set the current quark masses to zero. In that case, the QCD Lagrangian exhibits a flavor $S U(3)_{L} \times S U(3)_{R} \times U(1)_{L+R} \times U(1)_{L-R}$ symmetry. The vectorial $U(1)$ is related to the baryon number and the axial $U(1)$ is afflicted by the anomaly which gives the $\eta^{\prime}$ a mass of $1 \mathrm{GeV}$. The chiral group has therefore sixteen independent generators. This leads to sixteen conserved left- and right-handed currents,

$$
J_{\mu, A}^{a}=\bar{q}_{A} \gamma_{\mu} \frac{\lambda^{a}}{2} q_{A} \quad(a=1, \ldots, 8 ; A=L, R)
$$

or, equivalently, eight conserved vector and eight conserved axial-vector charges. These form an $\mathrm{SU}(3)$ algebra. However, this symmetry of the Lagrangian is not present in the ground state or the spectrum of the strongly interacting particles. Something very similar to the ferromagnet below the Curie temperature happens. The magnet Hamiltonian is rotationally invariant but below $T_{c}$ spontaneous magnetization sets in and all spins align in one direction breaking the symmetry of the Hamiltonian. Similarly in QCD only the vector charges are conserved allowing us to order the hadrons in multiplets of certain isospin and hypercharge quantum numbers. Nevertheless, the information about the axial charges is not lost. Goldstone's theorem [1] tells us that for any broken generator there exists a massless boson, in this case of pseudoscalar nature. This means that we should expect eight massless pseudoscalar mesons in the particle data listings. This is not the case. The fact of the matter is that the Goldstone bosons pick up a small mass related to the small current quark masses, like e.g.

$$
M_{\pi}^{2}=\left(m_{u}+m_{d}\right) B+O\left(\mathcal{M}^{2}\right)
$$

where $B=-<0|\bar{q} q| 0>/ F_{\pi}^{2}$ is the order parameter of the spontaneous symmetry breaking and $F_{\pi}$ measures the strength of the non-vanishing transition amplitude

$$
<0\left|A_{\mu}^{i}\right| \pi^{j}>=i \delta^{i j} p_{\mu} F_{\pi}
$$

In what follows, I will assume that $B \sim 1 \mathrm{GeV}$, i.e. that the quark mass expansion of the pseudoscalar meson masses is indeed governed by the leading term linear in the quark masses. It is interesting to observe that (2.4) gives us a recipe how to translate quark into meson masses. Evidently, at low energies the almost massless Goldstone bosons are the dominant degrees of freedom. This has been the theme of Heiri Leutwyler's lectures [2] two years ago and I refer the reader for a more detailed discussion of these underlying ideas. Also a recent review [3] is available which can be used as a first reading.

\subsection{Chiral Perturbation Theory (Mesons)}

The purest reaction of low-energy QCD is the elastic scattering of pions from pions in the threshold region. I will use this as an example to discuss the principles underlying the so-called chiral perturbation theory (CHPT). It is convenient to collect the Goldstone bosons in a matrix-valued field $U(x)=\exp \left(i \phi(x) / F_{\pi}\right)$, in the standard notation. To lowest order in the momenta and quark masses, the interactions of the Goldstone bosons e.g. can be described by the gauged non-linear $\sigma$-model, 


$$
\mathcal{L}^{(2)}=\frac{F^{2}}{4} \operatorname{Tr}\left[\nabla_{\mu} U^{\dagger} \nabla^{\mu} U\right]+\frac{F^{2} B}{4} \operatorname{Tr}\left[\mathcal{M}\left(U+U^{\dagger}\right)\right]
$$

with $\nabla_{\mu}$ a covariant derivative including the coupling to the external electroweak fields. The second term in (2.6) reflects the explicit symmetry breaking from the quark masses. Both terms are of chiral dimension two since they contain either two derivatives or one quark mass (which is equivalent to two derivatives, see (2.4)). To lowest order, the strong interactions are therefore given in terms of two parameters. These are $F$, the pion decay constant in the chiral limit, and $B$ related to the quark condensate (remember that I assume $B$ to be substantially larger than $F$ ). Calculating tree diagrams from this effective Lagrangian leads to the celebrated current algebra results. In particular, one recovers Weinberg's prediction for the $\pi \pi$ scattering amplitude, $A(s, t, u)=\left(s-M_{\pi}^{2}\right) / F_{\pi}^{2}$ [4]. Clearly, this smells like an expansion in energy since close to threshold, the c.m. energy $\sqrt{s}$ becomes very small and, consequently, the interaction between the Goldstone bosons weak. This is a particular imprint of the spontaneously broken chiral symmetry which allows for a "perturbative" expansion in the non-perturbative regime of QCD. Obviously, the effective Lagrangian (2.6) can not be the whole story. First, one would like to know how big the corrections from higher order terms (with more derivatives and/or quark mass insertions) are. Second, and more important, tree diagrams are always real. Twenty years ago at this school, Lehmann [5] demonstrated that unitarity forces one to consider loop diagrams. Based on the counting rules developed by Weinberg [6], which state that $n$-loop diagrams are suppressed by powers of $q^{2 n}$ (with $q$ a genuine small momentum), Gasser and Leutwyler [7] have systematized the whole approach for QCD. Let us consider the corrections which appear at next-to-leading order. First, there are the one-loop diagrams mandated by unitarity. Second, there are higher order contact terms with more derivatives or quark mass insertions. These are accompanied by a priori unknown coupling constants, the so-called low-energy constants. Their values can be fixed from phenomenology [7] or understood to a high degree of accuracy from the QCD version of the vector-meson dominance principle [8]. It is at this next-to-leading order where the various equivalent lowest order approaches differ. For example, the linear $\sigma$-model is a perfect candidate to be used instead of (2.6), but at next-to-leading order it simply gives low-energy constants at variance with the data [7]. Let me return to the example of $\pi \pi$ scattering. The amplitude expanded to one loop accuracy takes the form

$$
T_{\pi \pi}(s, t, u)=A(s, t, u)+B(s, t, u)+C(s, t, u),
$$

where $B(s, t, u)$ includes the loop (unitarity) effects and $C(s, t, u)$ the contribution from the higher dimensional operators. That this gives indeed a better description of the $\pi \pi$ phase shifts than the tree level prediction $A(s, t, u)$ can be seen in ref.[9]. In what follows, I will always confine myself to the one loop approximation. For a discussion about its range of applicability, see e.g. ref.[10]. 


\subsection{Inclusion of Matter Fields}

In this section I will be concerned with adding the low-lying spin-1/2 baryons $(N, \Sigma$, $\Lambda, \Xi)$ to the effective field theory. The inclusion of such matter fields is less straightforward since these particles are not related to the symmetry violation. However, their interactions with the Goldstone bosons is dictated by chiral symmetry. Let us denote by $B$ the conventional $3 \times 3$ matrix representing the spin- $1 / 2$ octet fields,

$$
B=\left(\begin{array}{ccc}
\frac{1}{\sqrt{2}} \Sigma^{0}+\frac{1}{\sqrt{6}} \Lambda & \Sigma^{+} & p \\
\Sigma^{-} & -\frac{1}{\sqrt{2}} \Sigma^{0}+\frac{1}{\sqrt{6}} \Lambda & n \\
\Xi^{-} & \Xi^{0} & -\frac{2}{\sqrt{6}} \Lambda
\end{array}\right)
$$

It is most convenient to choose a non-linear realization of the chiral symmetry [11] so that $B$ transforms as

$$
B \rightarrow K B K^{\dagger}
$$

where $K$ is a complicated function that does not only depend on the group elements $g_{L, R}$ of the $S U(3)_{L, R}$ but also on the Goldstone boson fields collected in $U(x)$, i.e. $K(x)=K\left(g_{L}, g_{R}, U(x)\right)$ defines a local transformation. Expanding $K$ in powers of the Goldstone boson fields, one realizes that a chiral transformation is linked to absorption or emission of pions, kaons and etas (which was the theme in the days of "current algebra" techniques). These topics are discussed in more detail in [3]. The lowest order meson-baryon Lagrangian is readily constructed. Let us restrict the discussion to processes with one incoming and one outgoing baryon, such as $\pi N$ scattering, threshold pion photoprodcution or baryon Compton scattering (otherwise, we would have to add contact $n$-fermion terms with $n \geq 4$ ). In that case, it takes the form

$$
\mathcal{L}_{\mathrm{MB}}^{(1)}=\operatorname{Tr}\left\{i \bar{B} \gamma^{\mu} D_{\mu} B-m_{0} \bar{B} B+\frac{1}{2} D \bar{B} \gamma^{\mu} \gamma_{5}\left\{u_{\mu}, B\right\}+\frac{1}{2} F \bar{B} \gamma^{\mu} \gamma_{5}\left[u_{\mu}, B\right]\right\}
$$

with $m_{0}$ the average octet mass (in the chiral limit) and $D_{\mu}$ the usual covariant derivative. There are two axial-vector type couplings multiplied by the conventional $F$ and $D$ constants. In the case of two flavors, there is only one such term proportional to $g_{A}=F+D$. Notice that the lowest order effective Lagrangian contains one derivative and therefore is of dimension one as indicated by the superscript '(1)'. In contrast to the meson sector (2.6), odd powers of the small momentum $q$ are allowed (thus, to leading order, no quark mass insertion appears since $\mathcal{M} \sim q^{2}$ ). It is instructive to expand (2.10) in powers of the Goldstone and external fields. From the vectorial term, one gets the minimal photon-baryon coupling, the two-Goldstone seagull (Weinberg term) and many others. Expansion of the axial-vectors leads to the pseudovector meson-baryon coupling, the celebrated Kroll-Rudermann term and much more. However, as already stated, it is not sufficient to calculate tree diagrams from the lowest order effective theory. In the presence of baryons, the loop expansion is more complicated than discussed in the previous section. First, since odd powers in $q$ are allowed, a one-loop calculation of order $q^{3}$ involves contact terms of dimension two and three, i.e. combinations of zero or one quark mass insertions with zero to three derivatives. These terms are collected in $\mathcal{L}_{\mathrm{MB}}^{(2,3)}$ and a complete list of them can be found in Krause's paper [12]. Second, the finiteness of the baryon mass in the chiral limit and that its value is comparable to the chiral symmetry breaking scale $\Lambda \sim M_{\rho}$ 
complicates the low energy structure. This has been discussed in detail by Gasser et al. [13]. Let me just give one illustrative example. The one loop contribution to the nucleon mass not only gives the celebrated non-analytic contribution proportional to $M_{\pi}^{3} \sim \mathcal{M}^{3 / 2}$ but also an infinite shift of $m_{0}$ which has to be compensated by a counterterm of dimension zero. It is a general feature that loops produce analytic contributions at orders below what one would naively expect (e.g. below $q^{3}$ from one loop diagrams). Therefore, in a CHPT calculation involving baryons one has to worry more about higher order contributions than it is the case in the meson sector. There is one way of curing this problem, namely to go into the extreme non-relativistic limit [14] and consider the baryons as very heavy (static) sources. Then, by a clever definition of velocity-dependent fields, one can eliminate the baryon mass term from the lowest order effective Lagrangian and expand all interaction vertices and baryon propagators in increasing powers of $1 / m_{0}$. This is similar to a Foldy-Wouthuysen transformation you all know from QED. In this limit one recovers a consistent derivative expansion. For example, the one loop contribution of the Goldstone bosons to the baryon self-energy is nothing but the non-analytic $M_{\phi}^{3}$ terms together with three contact terms from $\mathcal{L}_{\mathrm{MB}}^{(2)}$. However, one has to be somewhat careful still. The essence of the heavy mass formalism is that one works with old-fashioned time-ordered perturbation theory. So one has to watch out for the appearance of possible small energy denominators (infrared singularities). This problem has been addressed by Weinberg [15] in his discussion about the nature of the nuclear forces. The dangerous diagrams are the ones were cutting one pion line (this only concerns pions which are not in the asymptotic in- or out-states) separates the diagram into two disconnected pieces (one therefore speaks of reducible diagrams). These diagrams should be inserted in a Schrödinger equation or a relativistic generalization thereof with the irreducible ones entering as a potential. So the full CHPT machinery is applied to the irreducible diagrams. This should be kept in mind. For the purposes I am discussing, we do not need to worry about these complications. Being aware of them, it is then straightforward to apply baryon CHPT to many nuclear and particle physics problems $[3,16,17]$. I will illustrate this on two particular examples in the next sections. Before doing that, however, I would like to stress that all these calculations are only in their infancy. It is believed that for a good quantitative description one has to perform systematic calculations to order $q^{4}$, i.e. beyond next-to-leading order. There are many indications of this coming from the baryon masses, hyperon non-leptonic decays and so on. At present, only some rather unsystematic attempts have been done which incorporate some $q^{4}$ effects. In particular, it has been argued that one should include the low-lying spin-3/2 decuplet in the effective theory from the start $[16,18]$. A critical discussion of this approach can be found in ref.[19]. Clearly, more work is needed to clarify these issues. Let me now proceed and discuss two applications of the framework outlined here. These are calculations in flavor $S U(2)$ were the loop corrections are considerably smaller. As I will show, they shed some light on the non-perturbative structure of the nucleon. 


\subsection{Threshold Pion Photo- and Electroproduction}

Let us consider the reaction $\gamma(k)+N_{1}\left(p_{1}\right) \rightarrow \pi^{a}(q)+N_{2}\left(p_{2}\right)$ with $N_{1,2}$ denoting protons and/or neutrons and ' $a$ ' refers to the charge of the produced pion. In the case of real photons $\left(k^{2}=0\right)$ one talks of photoproduction whereas for virtual photons (radiated off an electron beam) the process is called electroproduction. Of particular interest is the threshold region where the photon has just enough energy to produce the pion at rest or with a very small three-momentum. In this kinematical regime, it is advantegous to perform a multipole decomposition since at threshold only the $S$-waves survive. These multipoles are labelled $E(M)_{l \pm}$, with $E(M)$ for electric (magnetic), $l=0,1,2, \ldots$ the pion orbital angular momentum and the \pm refers to the total angular momentum of the pion-nucleon system, $j=l \pm 1 / 2$. They parametrize the structure of the nucleon as probed with low energy photons. Let us first concentrate on the photoproduction case. At threshold, the differential cross section takes the form

$$
\left.\frac{|\mathbf{k}|}{|\mathbf{q}|} \frac{d \sigma}{d \Omega}\right|_{\mathbf{q} \rightarrow 0}=\left(E_{0+}\right)^{2}
$$

i.e. it is entirely given in terms of the electric dipole amplitude $E_{0+}$ (which at threshold is real). The quark mass expansion of $E_{0+}$ is given by

$$
E_{0+}=\frac{-i e}{8 \pi m} \frac{\mu(2+\mu)}{(1+\mu)^{3 / 2}} f\left(\bar{m} / \Lambda_{\mathrm{QCD}}\right)
$$

with $\mu=M_{\pi} / m \simeq 1 / 7$ and $\bar{m}$ the renormalization group invariant quark masses. Modulo logarithms, one can expand $f$ in powers of $\mu$,

$$
f=\frac{1}{\mu} f_{-1}+f_{0}+\mu f_{1}+\mathcal{O}\left(\mu^{2}\right)
$$

For charged pions $f_{-1}$ is non-vanishing and one is lead to the famous Kroll-Rudermann result [20] which states that $E_{0+}$ for $\gamma p \rightarrow \pi^{+} n$ and $\gamma n \rightarrow \pi^{-} p$ can be expressed in terms of the strong pion-nucleon coupling constant, the nucleon mass and some kinematical factors. Consequently, in these cases $E_{0+}$ is finite in the chiral limit. Matters are different for the case of neutral pions. Here, $f_{-1}$ vanishes and thus the leading term in the expansion of $E_{0+}$ goes like $\mu\left(\mu^{2}\right)$ for $\gamma p \rightarrow \pi^{0} p\left(\gamma n \rightarrow \pi^{0} n\right)$. The amplitude is therefore sensitive to the chiral symmetry breaking which makes it a good object to study experimentally and theoretically. If one performs a one loop CHPT calculation, one can get unambigously the next-to-leading order terms in the quark mass expansion of the eletric dipole amplitude. For the production of the proton, the threshold value is [21]

$$
E_{0+}=-\frac{e g_{\pi N}}{8 \pi m} \mu\left\{1-\left[\frac{1}{2}\left(3+\kappa_{p}\right)+\left(\frac{m}{4 F_{\pi}}\right)^{2}\right] \mu+\mathcal{O}\left(\mu^{2}\right)\right\},
$$

with $\kappa_{p}=3.71$ the anomalous magnetic moment of the proton. The second term in the square brackets is the new one found in the CHPT calculation. It stems from the so-called triangle diagram and its crossed partner. If one expresses $E_{0+}$ in terms of the conventional Lorentz invariant functions $A_{1,2,3,4}$, one finds that these diagrams give a contribution $\delta A_{1}=\left(e g_{\pi N} / 32 F_{\pi}^{2}\right) \mu$, i.e. they are non-analytic in the quark masses 
since $\mu \sim \sqrt{\hat{m}}$. Remember also that the electric dipole amplitude at threshold is proportional to $\mu A_{1}$ and therefore one gets this novel contribution at next-to-leading order in the quark mass expansion. Clearly, the expansion in $\mu$ is slowly converging, the coefficient of the term of order $\mu^{2}$ is so large that it compensates the leading term proportional to $\mu$. Therefore, for a meaningful prediction one has to go further in the expansion. This has been done in the relativistic formalism which sums up some of the higher order corrections [22]. One finds (at threshold) $E_{0+}=-1.33 \pm 0.09$ using the conventional units of $10^{-3} / M_{\pi^{+}}$. The error reflects solely the uncertainty in estimating the finite contact term contributing at order $q^{3}$. This is somewhat below the generally accepted experimental value of $-2.0 \pm 0.2$ (see e.g. Bernstein and Holstein [23] or Drechsel and Tiator [24] and references therein). However, one should point out that the calculation was performed in the isospin limit with $M_{\pi^{ \pm}}=M_{\pi^{0}}$ and $m_{p}=m_{n}$. It is a very tough problem to include isospin-breaking in a systematic fashion - I invite you to solve it. To get an idea about the effects related to it, one can perform a toy calculation and set the pion and nucleon masses by hand on their physical values in the various diagrams. Then, one finds $E_{0+}=-1.97$, which is an encouraging number but it has to be confirmed by a better calculation. The chief reason for the difference to the isospin-symmetric case is that the contribution of the triangle diagram now vanishes below the $\pi^{+} n$ threshold. It is furthermore interesting to observe that the one-loop corrections to the electric dipole amplitudes of charged pion photoproduction move the prediction closer to the data. These chiral corrections are suppressed by powers of $\mu^{2}$ and $\mu^{2} \ln \mu$ with respect to the leading Kroll-Ruderman term $\left(\sim \mu^{0}\right)$ and therefore a more accurate experimental determination of these quantities is called for. Let me now consider the electroproduction process. First, the low-energy theorems for the two $S$-wave multipoles $E_{0+}$ and $L_{0+}$ (for virtual photons, there is also a longitudinal coupling to the nucleon) have been discussed in the light of CHPT in ref.[25]. Let me stress that the LETs derived in refs.[21,25] are the ones implied by QCD and that they can be tested experimentally. Notice that there still seems to be some confusion concerning the meaning and the interpretation of these theorems [26]. However, I will not dwell on this topic here. Instead, I want to direct your attention to some new data. Welch et al.[27] have published the $S$-wave cross section for the reaction $\gamma^{\star} p \rightarrow \pi^{0} p$ very close to the photon point. This measurement is a quantum step compared to previous determinations which mostly date back to the seventies when pion electroproduction was still a hot topic in particle physics. In this experiment, $k^{2}$ varied between -0.04 and $-0.10 \mathrm{GeV}^{-2}$ and the $S$-wave cross section could be extracted with an unprecedented accuracy (see fig.2 in [27]). This is also the kinematical regime where a CHPT calculation might offer some insight. Indeed, in ref.[28] it was shown that the $k^{2}$-dependence of this cross section seems to indicate the necessity of loop effects. With conventional models including e.g. form factors and the anomalous magnetic moment coupling the trend of the data can not be described. However, the corrections from the one loop diagrams to the tree level prediction are substantial. This gives further credit to the previously made statement that a calculation beyond next-to-leading order should be performed. The last topic I want to address in this section concerns the determination of the nucleon axial radius from charged pion electroproduction. Let me briefly explain how the axial form factor comes into play. The basic matrix element to be considered is the time-ordered product of the electromagnetic (vector) current with the interpolating pion field sandwiched 
between nucleon states. Now one can use the PCAC relation and express the pion field in terms of the divergence of the axial current. Thus, a commutator of the form $[\mathrm{V}, \mathrm{A}]$ arises. Current algebra tells us that this gives an axial current between the incoming and outgoing nucleon fields and, alas, the axial form factor. The isospin factors combine in a way that they form a totally antisymmetric combination which can not be probed in neutral pion production. These ideas were formalized in the venerable low-energy theorem (LET) due to Nambu, Lurié and Shrauner [29] for the isospin-odd electric dipole amplitude $E_{0+}^{(-)}$in the chiral limit,

$$
E_{0+}^{(-)}\left(M_{\pi}=0, k^{2}\right)=\frac{e g_{A}}{8 \pi F_{\pi}}\left\{1+\frac{k^{2}}{6} r_{A}^{2}+\frac{k^{2}}{4 m^{2}}\left(\kappa_{V}+\frac{1}{2}\right)+\mathcal{O}\left(k^{3}\right)\right\}
$$

Therefore, measuring the reactions $\gamma^{\star} p \rightarrow \pi^{+} n$ and $\gamma^{\star} n \rightarrow \pi^{-} p$ allows to extract $E_{0+}^{(-)}$ and one can determine the axial radius of the nucleon, $r_{A}$. This quantity measures the distribution of spin and isospin in the nucleon, i.e. probes the Gamov-Teller operator $\sigma \cdot \tau$. A priori, the axial radius is expected to be different from the typical electromagnetic size, $r_{\mathrm{em}} \simeq 0.85 \mathrm{fm}$. It is customary to parametrize the axial form factor $G_{A}\left(k^{2}\right)$ by a dipole form, $G_{A}\left(k^{2}\right)=\left(1-k^{2} / M_{A}^{2}\right)^{-2}$ which leads to the relation $r_{A}=\sqrt{12} / M_{A}$. The axial radius determined from electroproduction data is typically $r_{A}=0.59 \pm 0.04 \mathrm{fm}$ whereas (anti)neutrino-nucleon reactions lead to somewhat larger values, $r_{A}=0.65 \pm 0.03 \mathrm{fm}$. This discrepancy is usually not taken seriously since the values overlap within the error bars. However, it was shown in ref.[30] that pion loops modify the LET (2.15) at order $k^{2}$ for finite pion mass. In the heavy mass formalism, the coefficient of the $k^{2}$ term reads

$$
\frac{1}{6} r_{A}^{2}+\frac{1}{4 m^{2}}\left(\kappa_{V}+\frac{1}{2}\right)+\frac{1}{128 F_{\pi}^{2}}\left(1-\frac{12}{\pi^{2}}\right)
$$

where the last term in (2.16) is the new one. This means that previously one had extracted a modified radius, the correction being $3\left(1-12 / \pi^{2}\right) / 64 F_{\pi}^{2} \simeq-0.046 \mathrm{fm}^{2}$. This closes the gap between the values of $r_{A}$ extracted from electroproduction and neutrino data. It remains to be seen how the $1 / m$ suppressed terms will modify the result (2.16). Such investigations are underway.

\subsection{Nucleon Compton Scattering}

Consider low-energy (real) photons scattering off a spin- $1 / 2$ particle. In forward direction, the scattering amplitude takes the form

$$
T_{1 / 2}(\omega)=f_{1}\left(\omega^{2}\right) \epsilon_{f}^{*} \cdot \epsilon_{i}+i \omega f_{2}\left(\omega^{2}\right) \sigma \cdot\left(\epsilon_{f}^{*} \times \epsilon_{i}\right)
$$

where $\omega$ is the photon frequency and the $\epsilon_{i, f}$ are the polarization vectors in the initial and final state, respectively. The energy expansion of the spin-dependent amplitude $f_{1}\left(\omega^{2}\right)$ reads

$$
f_{1}\left(\omega^{2}\right)=-\frac{e^{2} Z^{2}}{4 \pi m}+(\alpha+\beta) \omega^{2}+\mathcal{O}\left(\omega^{4}\right)
$$

where the first energy-independent term is nothing but the Thomson amplitude mandated by gauge invariance. Therefore, to leading order, the photon only probes some 
global properties like the mass or electric charge of the spin- $1 / 2$ target. At next-toleading order, the non-perturbative structure is parametrized by two constants, the so-called electric and magnetic polarizabilities (more correctly, one might want to talk about "magnetic susceptibility", however, I will use the common language). A detailed discussion of the CHPT calculation of these fundamental two-photon observables is given in ref.[3] (see also the references quoted there). Here, I just want to add a few educational remarks. For the proton, the Thomson term is non-vanishing and it is interesting to compare its magnitude to the one from the polarizability contribution using $(\alpha+\beta)_{p}=14.2 \cdot 10^{-4} \mathrm{fm}^{3}$. The recent Illinois Compton scattering experiment [31] to determine $\alpha_{p}$ and $\beta_{p}$ was performed at photon energies ranging from 32 to $72 \mathrm{MeV}$. While at the lowest $\omega$ the polarizability term amounts to a 31 per cent correction to the Thomson term contribution $\left(=-1.22 \cdot 10^{-4} \mathrm{fm}\right)$, at 72 $\mathrm{MeV}$ the second term in the energy expansion is 1.5 times as large as the leading term. Clearly, higher order $\left(\omega^{4}\right)$ contributions have to be taken into account at such energies. Another remark concerns the behaviour of $\alpha_{p, n}$ and $\beta_{p, n}$ in the chiral limit. These quantities diverge as $1 / M_{\pi}$ as the pion mass tends to zero. This is expected since the two photons probe the long-ranged pion cloud, i.e. there is no more Yukawa suppression as in the case for a finite pion mass. Now a well-known dispersion sum rule relates $(\alpha+\beta)$ to the total nucleon photoabsorption cross section. The latter is, of course, also well-behaved in the chiral limit which at first sight seems to be at variance with the behaviour of the expansion of the scattering amplitude. But be aware that the general form of (2.18) has been derived under the assumption that there is a well defined low-energy limit. Similar observations can also be made concerning the chiral expansion of the $\pi \pi$ scattering amplitude discussed in section 2. Let me now focus on the spin-dependent amplitude $f_{2}\left(\omega^{2}\right)$ which has an expansion analogous to (2.18),

$$
f_{2}\left(\omega^{2}\right)=f_{2}(0)+\gamma \omega^{2}+\mathcal{O}\left(\omega^{4}\right)
$$

By the optical theorem, the imaginary part of $f_{2}$ is related to the photoabsorption cross sections for circularly polarized photons on polarized nucleons,

$$
\operatorname{Im} f_{2}\left(\omega^{2}\right)=-\frac{1}{8 \pi}\left[\sigma_{3 / 2}(\omega)-\sigma_{1 / 2}(\omega)\right]
$$

where the indices $3 / 2$ and $1 / 2$ refer to the total $\gamma N$ helicities. We will come back to this relation later on. Let me now turn to the discussion of the Taylor coefficients $f_{2}(0)$ and $\gamma$ in eq.(2.19). There exists a celebrated LET for $f_{2}(0)$ due to Low, GellMann and Goldberger [32]. Using very general principles like gauge invariance, Lorentz invariance and crossing symmetry, they showed that

$$
f_{2}(0)=-\frac{e^{2} \kappa^{2}}{8 \pi m^{2}} .
$$

Here, $\kappa$ denotes the anomalous magnetic moment of the particle the photon scatters off. In CHPT, one can easily derive the LET making use of the heavy baryon formalism as shown in ref.[33]. It stems from some contact terms which are nominally suppressed by powers of $1 / \mathrm{m}$. To one-loop order, one can give $f_{2}\left(\omega^{2}\right)$ in closed form since only a few loop diagrams contribute (in addition to the contact terms giving the LET), 


$$
f_{2}\left(\omega^{2}\right)=-\frac{e^{2} \kappa^{2}}{8 \pi m^{2}}+\frac{e^{2} g_{A}^{2}}{32 \pi^{3} F^{2}}\left[\frac{M^{2}}{\omega^{2}} \arcsin ^{2}\left(\frac{\omega}{M}\right)-1\right]
$$

From this the slope parameter $\gamma$ follows immediately. Notice that $\gamma$ is again the sum of an electric and magnetic piece, but in the absence of any data, I will only discuss the sum. The em decomposition can be supplied upon request. To leading order, $\gamma$ is identical for the $\mathrm{p}$ and the $\mathrm{n}$,

$$
\gamma=\frac{e^{2} g_{A}^{2}}{96 \pi^{3} F_{\pi}^{2} M_{\pi}^{2}}=4.4 \cdot 10^{-4} \mathrm{fm}^{4} .
$$

In ref.[33] it was shown that including $1 / \mathrm{m}$ suppressed effects in the relativistic formalism together with an estimate of the $\Delta(1232)$ resonance contribution leads to $\gamma_{p}=-1.5$ and $\gamma_{n}=-0.5$ (in conventional units), in fair agreement with the estimate based on the dispersion sum rule

$$
\gamma=-\frac{1}{4 \pi^{2}} \int_{M_{\pi}}^{\infty} \frac{d \omega}{\omega^{2}}\left[\sigma_{3 / 2}(\omega)-\sigma_{1 / 2}(\omega)\right]
$$

which follows directly from eq.(2.20). Using the helicity cross sections provided by Workman and Arndt [34] one gets $\gamma_{p}^{\mathrm{emp}}=-0.89$ and $\gamma_{n}^{\mathrm{emp}}=-0.53$. Clearly, a direct experimental determination of these observables is called for. To give an idea about the size of the effect induced by the "spin-polarizability" $\gamma$, let us compare the leading order term $f_{2}(0)=-5.19 \cdot 10^{-4} \mathrm{fm}^{2}$ with the correction from $\gamma \omega^{2}$ at various energies. For $\omega=50 \mathrm{MeV}$, this term amounts to a 6 per cent reduction of the leading one. At $100 \mathrm{MeV}$ photon energy, the correction has grown to 22 per cent. For comparison, evaluating the full one-loop amplitude (2.22), one finds corrections of 7 and 37 percent for $\omega=50$ and $100 \mathrm{MeV}$, respectively. These latter numbers will, of course, be changed by higher loop corrections. We notice that the influence of the next-to-leading order term is much less pronounced than in the case of the spin-independent amplitude and thus an accurate determination of $\gamma$ and its electric and magnetic components will be more difficult. The last topic I want to address in this section is the so-called DrellHearn-Gerasimov [35] sum rule and its extension to virtual photons. For photons with four-momentum $k^{2} \leq 0$ the extended DHG sum rule takes the form

$$
I\left(k^{2}\right)=\int_{\omega_{\mathrm{thr}}}^{\infty} \frac{d \omega}{\omega}\left[\sigma_{1 / 2}\left(\omega, k^{2}\right)-\sigma_{3 / 2}\left(\omega, k^{2}\right)\right]
$$

For real photons, this reduces to the DHG sum rule, which has not yet been tested experimentally. For the proton, one has $I(0)=-\pi e^{2} \kappa_{p}^{2} / 2 m^{2}=-0.53 \mathrm{GeV}^{-2}$. At large $k^{2} \simeq-10 \mathrm{GeV}^{2}$, the EMC [36] data tell us that $I$ is positive and thus has to undergo a sign change. CHPT allows one to calculate the slope of $I\left(k^{2}\right)$ in the vicinity of the photon point [37]. While the most recent phenomenological analysis gives a kink structure at small $k^{2}$ [38], in CHPT one finds that $I\left(k^{2}\right)$ increases monotonically. At present, one can not decide upon this fine detail but has to wait for the experimental determinations which are possible with CW machines and are planned (MAMI, CEBAF, ...). Finally, I wish to stress again that CHPT allows to systematically explore the consequences of the spontaneously broken chiral symmetry of QCD. It is not a model as should have become clear from the above discussion but rather an exact non-perturbative method. 


\section{The Quark Structure of the Nucleon}

In this section I will be concerned with the quark structure of the nucleon as revealed from measurements using electroweak probes. In particular, one can address the questions surrounding the possible admixtures of strange quark components into the proton's wave function. Since these are mostly small effects, I will also discuss the effects of heavy quark and radiative corrections to the processes which allow one to determine the electroweak form factors.

\subsection{Currents, Sizes and Form Factors of the Nucleon}

The starting point of the discussion are the well-known electromagnetic and axial currents based on photon and charged vector boson exchanges. Let us first consider the vector current $J_{\mu}$ stemming from the one-photon exchange. Between nucleon states of momentum $p$ and helicity $s$ (in general, I will suppress helicitiy indices) Lorentz-invariance, parity and charge conjugation allow us to write

$$
<N\left(p^{\prime}, s^{\prime}\right)\left|J_{\mu}\right| N(p, s)>=e \bar{u}\left(p^{\prime}, s^{\prime}\right)\left[F_{1}\left(q^{2}\right) \gamma_{\mu}+F_{2}\left(q^{2}\right) \frac{i \sigma_{\mu \nu} q^{\nu}}{2 m}\right] u(p, s)
$$

with $q_{\mu}=\left(p^{\prime}-p\right)_{\mu}$ the four-momentum transfer, $m$ denotes the nucleon mass and $u(p, s)$ a conventional nucleon spinor. The finite extension (non-perturbative structure) is parametrized in terms of the Dirac and Pauli form factors (ffs), $F_{1}\left(q^{2}\right)$ and $F_{2}\left(q^{2}\right)$, respectively. Gauge invariance of the electromagnetic interactions demands $\partial_{\mu} J^{\mu}(x)=0$. The physical interpretation of these ffs is particularly transparent in the Breit (brick wall) frame, in which the photon transfers no energy, $q_{\mu}=(0, \mathbf{q})$. The time component of $J_{\mu}$ is the charge density, $J_{0}=\rho$ and the space components lead to the distribution of magnetism,

$$
\begin{aligned}
<N\left(\mathbf{q} / 2, s^{\prime}\right)\left|J_{0}\right| N(-\mathbf{q} / 2, s)> & =e G_{E}\left(q^{2}\right) \delta_{s^{\prime} s} \\
& =e\left[F_{1}\left(q^{2}\right)-\tau F_{2}\left(q^{2}\right)\right] \delta_{s^{\prime} s} \\
<N\left(\mathbf{q} / 2, s^{\prime}\right)|\mathbf{J}|(-\mathbf{q} / 2, s)> & =\frac{e}{2 m} G_{M}\left(q^{2}\right) \chi_{s^{\prime}}^{\dagger} i \sigma \times \mathbf{q} \chi_{s} \\
& =\frac{e}{2 m}\left[F_{1}\left(q^{2}\right)+F_{2}\left(q^{2}\right)\right] \chi_{s^{\prime}}^{\dagger} i \sigma \times \mathbf{q} \chi_{s}
\end{aligned}
$$

with $\chi_{s}$ a two-component spinor and $\tau=-q^{2} / 4 m^{2}=Q^{2} / 4 m^{2}\left(Q^{2}>0\right.$ for space-like photons). Fourier-transformation of the electric charge density leads to

$$
\begin{aligned}
\int \rho(\mathbf{r}) j_{0}(q r) d^{3} r & =\int \rho(\mathbf{r}) d^{3} r-\frac{1}{6} Q^{2} \int \rho(\mathbf{r}) r^{2} d^{3} r+\ldots \\
& =Z-\frac{1}{6} Q^{2}<r^{2}>_{E}+\ldots
\end{aligned}
$$

where the first term is obviously the electric charge of the particle (here, the proton or the neutron) and the second one defines the electric charge radius. It is conventional to normalize these charge radii via

$$
<r^{2}>=\left.\frac{6}{G(0)} \frac{d G\left(q^{2}\right)}{d q^{2}}\right|_{q^{2}=0}
$$


with the exception of the neutron charge radius since in that case $G(0)=0$ so that $\left\langle r_{E}^{2}\right\rangle_{n}$ is simply six times the slope of the neutron form factor. The electric (E) and magnetic (M) ffs are normalized as follows

$$
G_{E}^{p}(0)=1, \quad G_{E}^{n}(0)=0, \quad G_{M}^{p}(0)=\mu_{p}=2.793, \quad G_{M}^{n}(0)=\mu_{n}=-1.913 .
$$

For the various radii, the presently available data are [39]

$$
\begin{aligned}
& <r_{E}^{2}>_{p}^{1 / 2}=0.86 \pm 0.01 \mathrm{fm}, \quad<r_{M}^{2}>_{p}^{1 / 2}=0.86 \pm 0.06 \mathrm{fm}, \\
& <r_{E}^{2}>_{n}=-0.12 \pm 0.01 \mathrm{fm}^{2}, \quad<r_{M}^{2}>_{n}^{1 / 2}=0.88 \pm 0.07 \mathrm{fm} .
\end{aligned}
$$

A few remarks on these numbers are in order. First, the typical electromagnetic (em) size is $r_{\mathrm{em}} \simeq 0.85 \mathrm{fm}$ and the negative value of the neutron charge radius squared shows that a surplus of negative charge in the exterior region of the neutron must exist. Most disturbing is the fact that in particular the magnetic charge radius of the neutron, which is a quantity as fundamental as say the magnetic moment, is only poorly known. As pointed out by Arenhövel and Schoch at this school [40] experiments are under way to remedy the situation. At larger momentum transfers, it has become common to use the so-called dipole fits, which proved to be embarrasingly good up to several $\mathrm{GeV}^{2}$ on typical logarithmic plots when elastic electron-proton scattering experiments were performed in the sixties and early seventies as discussed by Taylor here [41]. The dipole fit reads

$$
G_{E}^{p}\left(Q^{2}\right)=\frac{G_{M}^{p}\left(Q^{2}\right)}{\mu_{p}}=\frac{G_{M}^{n}\left(Q^{2}\right)}{\mu_{n}}=G_{D}\left(Q^{2}\right)=\frac{1}{\left(1+Q^{2} / 0.71 \mathrm{GeV}^{2}\right)^{2}} .
$$

For the electric ff of the neutron, the "trivial" dipole fit is $G_{E}^{n}\left(Q^{2}\right)=0$, however, in nature this $\mathrm{ff}$ is small but non-vanishing. It can be approximated by [42]

$$
G_{E}^{n}\left(Q^{2}\right)=-\frac{\mu_{n} \tau}{1+Q^{2} / 0.42 \mathrm{GeV}^{2}} G_{D}\left(Q^{2}\right) .
$$

In refs.[43], you can take a look at the quality of these fits on a linear scale for $Q^{2}$ up to $4 \mathrm{GeV}^{2}$ - it is obvious that the dipole fit is at most a fair approximation. Also, it has never been given a sound theoretical foundation. While the dipole mass $M_{D}=0.843$ $\mathrm{GeV}$ is suggestive of vector meson exchange (like in the vector meson dominance approach to photon-hadron couplings), such a mechanism would naturally lead to a monopole fall-off. In case of the neutron electric ff, which is determined indirectly from elastic scattering off the deuteron and subtraction of the proton ff, a major uncertainty stems from the use of the underlying model of the nucleon-nucleon force as discussed in ref.[42]. Here, experiments using polarized electrons and polarized helium targets are hoped to improve the theoretical uncertainties. Definitively, we need a better determination of these fundamental nucleon properties. It is also instructive to see what perturbative QCD tells us about the large $Q^{2}$ behaviour of these ffs. Based on the quark counting rules [44], which state that to distribute the large photon momentum equally to three quarks in the nucleon, two gluon exchanges are necessary, one finds that $F_{1}\left(Q^{2}\right) \sim 1 / Q^{4}$ and $F_{2}\left(Q^{2}\right) \sim 1 / Q^{6}$ since the gluon propagator goes like $1 / Q^{2}$ and in the case of $F_{2}$ an extra $1 / Q^{2}$ is needed for the helicity flip. At which value of $Q^{2}$ this asymptotic behaviour sets in is not known, certainly way beyond $1 \mathrm{GeV}^{2}$ as 
originaly thought. Notice that the dipole fits to the nucleon ffs have the correct large $Q^{2}$ behaviour. This is a further mystery surrounding these fits. It might be based on the fact that a simple meson-cloud model of the nucleon also leads to such fall-offs (in the one-boson exchange approximation). Let me now switch to the axial current (related to the exchange of charged vector bosons). Its nucleon matrix element reads

$$
<N\left(p^{\prime}\right)\left|A_{i}^{\mu}(0)\right| N(p)>=\bar{u}\left(p^{\prime}\right)\left[G_{A}\left(q^{2}\right) \gamma^{\mu}+\frac{G_{P}\left(q^{2}\right)}{2 m} q^{\mu}\right] \gamma_{5} \frac{\tau_{i}}{2} u(p)
$$

where $G_{A}\left(q^{2}\right)$ is the axial ff. The induced pseudoscalar ff $G_{P}\left(q^{2}\right)$ is essentially pole dominated and only accessible via scattering processes involving heavy leptons (like e.g. muons). I will not consider it in what follows. $G_{A}(0)$ is nothing but the axialvector coupling constant measured in neutron $\beta$-decay, $g_{A}=1$.26. The $Q^{2}$-dependence of $G_{A}\left(Q^{2}\right)$ follows again a dipole form,

$$
G_{A}\left(Q^{2}\right)=\frac{g_{A}}{\left(1+Q^{2} / M_{A}^{2}\right)^{2}}
$$

The value of the axial cut off mass was already discussed in section 2.4, it translates into a typical axial size of $r_{A} \simeq 0.65 \mathrm{fm}$. This is considerably smaller than the typical em size. Therefore, the size of the nucleon depends on the probe one uses. In fact, the hierachy of these various nucleon sizes can nicely be understood in the topological soliton model of the nucleon as spelled out in detail in ref.[45]. In a nutshell, the argument goes as follows. The isoscalar baryon number current, whose space-integral is the baryon number, is given by the pion fields in the soliton with an extension of $r_{B}=0.5 \mathrm{fm}$. The isoscalar photon, however, sees in addition the virtual $\omega$-meson content of the nucleon. This leads to an isoscalar charge radius of $\left\langle r_{E}^{2}>_{I=0}=\right.$ $r_{B}^{2}+6 / M_{\omega}^{2}=(0.8 \mathrm{fm})^{2}$ in nice agreement with the data. By a similar argument, one expects a somewhat smaller axial radius since the additional factor now carries the axial vector mass of $1.1 \mathrm{GeV}$ and one is thus naturally lead to an axial extension of $0.65 \mathrm{fm}$.

\subsection{A Toy Model: Quark Distributions from Nucleon Form Factors}

To understand how the diferent electroweak properties as parametrized by the various ffs can give insight into the quark structure of the nucleon in the non-perturbative regime, let us consider a simple (too simple) model. The proton and the neutron are made up of valence $u$ and $d$ quarks and a sea of quark-antiquark pairs. We assume that the $\mathrm{p}$ and the $\mathrm{n}$ are simply related by the interchange of one valence $\mathrm{u}$ with one valence d quark. For the sake of simplicity, let us forget the gluons and just imagine some confinement mechanism which keeps the current quarks within the typical hadronic volume of $1 \mathrm{fm}^{3}$. Denoting by $J_{\mu}^{j}$ the em current associated to the quarks of flavor $j=u, d, s, c, b, t$, we can write [46]

$$
\begin{aligned}
<N\left(p^{\prime}, s^{\prime}\right)\left|J_{\mu}\right| N(p, s)> & =<N\left(p^{\prime}, s^{\prime}\right)\left|\sum_{j} J_{\mu}^{j}\right| N(p, s)> \\
& =e \sum_{j} \bar{u}\left(p^{\prime}, s^{\prime}\right) Q_{j}\left(\gamma_{\mu} F_{1}^{j}+\frac{i \sigma_{\mu \nu} q^{\nu}}{2 m} F_{2}^{j}\right) u(p, s)
\end{aligned}
$$


where $Q_{j}$ denotes the quark charges and the quarks are considered as point-like Dirac particles with no anomalous magnetic moment. In terms of the electric and magnetic ffs, this decomposition takes the form

$$
G_{E, M}^{(p, n)}=\sum_{j} Q_{j} G_{E, M}^{j(p, n)}
$$

These expressions are exact under the assumptions made as long as the sum extends over all quark flavors. Furthermore, the $G^{j}$ contain contributions from quarks as well as from antiquarks. Therefore, the $q \bar{q}$ sea does not contribute to the total charge since $Q_{j}=-\bar{Q}_{j}$. Since the $\mathrm{p}$ and the $\mathrm{n}$ as well as the $\mathrm{u}$ and the $\mathrm{d}$ quark are considered to be essentially the same particles differing only by an isospin rotation, it should be possible to separate the response of the various quark flavors [47]. For doing that, one has to make further assumptions. First, one postulates a local isospin invariance of the type $J_{\mu}^{u, p}(x)=J_{\mu}^{d, n}(x)$ and $J_{\mu}^{d, p}(x)=J_{\mu}^{u, n}(x)$ (which has never been tested and is doubtful in the light of the recent structure function measurements). Second, we assume that all other quark flavors do not contribute, i.e. $J_{\mu}^{q, p}(x)=J_{\mu}^{q, n}(x)=0$ for $q=s, c, b, t$. This allows to uniquely give the em $\mathrm{u}$ and d distributions in terms of appropriate combinations of the em ffs of the proton and the neutron. The final formulae are obtained by normalizing with respect to the total quark charges, e.g. pulling out a factor $4 / 3$ for the $u$ quark distribution in the proton. This leads to [48]

$$
\begin{aligned}
G_{E, M}^{u} & =G_{E, M}^{p}+\frac{1}{2} G_{E, M}^{n}, \\
G_{E, M}^{d} & =G_{E, M}^{p}+2 G_{E, M}^{n} .
\end{aligned}
$$

It is instructive to get some numbers from eq.(3.12). First, let us stick in the magnetic moments. We find $G_{M}^{u}(0)=1.836$ and $G_{M}^{d}(0)=-1.033$, which is not very different from the $S U(3)$ result for constituent quarks. Notice, however, that here we are considering current quarks. The implications of this result are discussed in more detail in Beck's lecture [48]. Similarly, for the electric charge radii one finds $\left.<r_{E}^{2}\right\rangle_{u}=0.68 \pm 0.02 \mathrm{fm}^{2}$ and $\left\langle r_{E}^{2}\right\rangle_{d}=0.51 \pm 0.02 \mathrm{fm}^{2}$ which is a 35 per cent difference. To further separate the valence from the sea quarks, one needs additional assumptions about the radial distributions as detailed in ref.[48]. In any case, this discussion should only be considered illustrative, the model is too crude to attach a deep physical significance to these numbers. However, in a very simple fashion it demonstrates how a clever combination of measurable hadron form factors allows us to make statements about quark distributions. This problem will now be tackled in a more serious fashion.

\subsection{Electroweak Currents}

In this section, I will define the electroweak currents related to the exchanges of the various vector bosons of the minimal standard model. These spin- 1 particles are the massless photon and the massive $W^{ \pm}$and $Z^{0}$ bosons. At tree level, the couplings of these to the various quark flavors are specified completely in terms of the $U(1)$ gauge coupling $g$ and the $S U(2)$ gauge coupling $g^{\prime}$. Equivalently, one can use the em coupling $e$ and weak mixing angle $\theta_{W}$ since $g=e \sin \theta_{W}=e s$ and $g^{\prime}=g / 4 \cos \theta_{W}=g / 4 c$. 
Also, the vector boson masses are related at tree level via $M_{Z}=M_{W} / c$. For the most recent values, see Altarelli's lectures at this school [49]. Let us consider again the photon current which is purely vectorial and its couplings to the light quarks $\mathrm{u}$, $\mathrm{d}$ and s. One has

$$
\begin{aligned}
J_{\mu}^{\gamma} & =\sum_{q=u, d, s} Q_{q} \bar{q} \gamma_{\mu} q=\frac{2}{3}\left(\bar{u} \gamma_{\mu} u\right)-\frac{1}{3}\left(\bar{d} \gamma_{\mu} d+\bar{s} \gamma_{\mu} s\right) \\
& =\sum_{q=u, d, s} \bar{q} \gamma_{\mu} \frac{1}{2}\left(\lambda^{3}+\frac{1}{\sqrt{3}} \lambda^{8}\right) q \\
& =\frac{1}{2}\left(\bar{u} \gamma_{\mu} u-\bar{d} \gamma_{\mu} d\right)+\frac{1}{6}\left(\bar{u} \gamma_{\mu} u+\bar{d} \gamma_{\mu} d-2 \bar{s} \gamma_{\mu} s\right) .
\end{aligned}
$$

I have exhibited the two most frequently used conventions. In the second line, the decomposition in terms of Gell-Mann's flavor $S U(3)$ matrices is shown. One notices that the photon is blind to the singlet piece $\sim \lambda^{0}$. In the third line, the photon current is decomposed into its isovector (IV) and isoscalar (IS) components. This current leads to the em form factors $G_{E, M}^{\gamma}$ or $F_{1,2}^{\gamma}$ discussed before (the label ' $\gamma$ ' is needed to differentiate these ffs from the ones related to the weak neutral current as discussed below). The charged weak currents are flavor-changing and lead to the isovector axial ff discussed before. Further information comes from the weak neutral current mediated by the $Z^{0}$. Its coupling to the quark has a vectorial and an axialvector piece. Ignoring the overall coupling strength $g^{\prime}$ and defining $V_{q}=\bar{q} \gamma_{\mu} q$ for a quark of flavor $q$, the vector part at tree level reads

$$
\begin{aligned}
V_{\mu}^{Z} & =\left(\frac{1}{4}-\frac{2}{3} s^{2}\right) V_{u}+\left(-\frac{1}{4}+\frac{1}{3} s^{2}\right)\left(V_{d}+V_{s}\right) \\
& =\frac{1}{2}\left(\frac{1}{2}-s^{2}\right)\left(V_{u}-V_{d}\right)+\frac{1}{6}\left(\frac{1}{2}-s^{2}\right)\left(V_{u}+V_{d}-2 V_{s}\right)-\frac{1}{12}\left(V_{u}+V_{d}+V_{s}\right) \\
& =\left(\frac{1}{2}-s^{2}\right) \frac{1}{2}\left(V_{u}-V_{d}\right)+\frac{1}{6} s^{2}\left(V_{u}+V_{d}-2 V_{s}\right)-\frac{1}{4} V_{s}
\end{aligned}
$$

where again the second line gives the $S U(3)$ components and the third line the decomposition into IS, IV and explicit strange pieces. Similarly, with $A_{q}=\bar{q} \gamma_{\mu} \gamma_{5} q$ we have for the axial-vector current

$$
\begin{aligned}
A_{\mu}^{Z} & =-\frac{1}{4} A_{u}+\frac{1}{4}\left(A_{d}+A_{s}\right) \\
& =-\frac{1}{4}\left(A_{u}-A_{d}\right)-\frac{1}{12}\left(A_{u}+A_{d}-2 A_{s}\right)+\frac{1}{12}\left(A_{u}+A_{d}+A_{s}\right) \\
& =-\frac{1}{2}\left[\frac{1}{2}\left(A_{u}-A_{d}\right)\right]+\frac{1}{4} A_{s}
\end{aligned}
$$

Two remarks are in order. First, the $Z^{0}$ couples to the singlet component $\sim \lambda^{0} \sim$ $(u+d+s)$ and, second, there is no isoscalar axial coupling at tree level. For the following discussion, let me express the ffs related to the $Z^{0}$-exchange in the flavor $S U(3)$ basis following Kaplan and Manohar [50], 


$$
\begin{aligned}
F_{1,2}^{Z} & =\sum_{\alpha=0,3,8}\left(b_{\alpha}-a_{\alpha} s^{2}\right) F_{1,2}^{\alpha} ; \quad F_{1,2}^{\gamma}=\sum_{\alpha=0,3,8} a_{\alpha} F_{1,2}^{\alpha}, \\
G_{1} & =-\sum_{\alpha=0,3,8} b_{\alpha} G_{A}^{\alpha}=-\sum_{\alpha=0,3,8} b_{\alpha} G_{1}^{\alpha}, \\
a^{0} & =0, \quad a^{3}=1, \quad a^{8}=\frac{1}{\sqrt{3}} ; \quad b^{0}=-\frac{1}{4}, \quad b^{3}=\frac{1}{2}, \quad b^{8}=\frac{1}{2 \sqrt{3}} .
\end{aligned}
$$

The value of the a's and b's are subject to radiative and heavy quark corrections as discussed below. For completeness, I have also given the em ffs in this basis. Using for the $S U(3)$ generators $\operatorname{Tr}\left(T^{a} T^{b}\right)=\delta^{a b} / 2$ and supplying the isosinglet currents with an overall factor $1 / 3$, the normalizations of the various ffs are given as follows. From the proton and the neutron charge, we have

$$
F_{1}^{3}(0)=1 / 2, \quad F_{1}^{8}(0)=\sqrt{3} / 2
$$

Similarly, the $\mathrm{p}$ and $\mathrm{n}$ anomalous magnetic moments lead to

$$
F_{2}^{3}(0)=\left(\kappa_{p}-\kappa_{n}\right) / 2, \quad F_{2}^{8}(0)=\sqrt{3}\left(\kappa_{p}+\kappa_{n}\right) / 2 .
$$

$F_{1}^{0}(0)$ is equal to unity since it gives the baryon number. However, this does not exclude a finite extension of $s \bar{s}$ pairs leading to a strange electric radius (in complete analogy to the neutron charge radius). The value of the singlet anomalous magnetic moment is not known. If there are no strange quarks in the proton, one has $F_{2}^{0}(0)=$ $\kappa_{p}+\kappa_{n}=-0.12$. So a measurement of $F_{2}^{0}(0)$ will give information about the possible strange quark contribution to the anomalous magnetic moment, $F_{2}^{0}(0)=\kappa_{p}+\kappa_{n}+\mu_{s}$. The triplet and octet normalizations of the axial ff are given by

$$
G_{1}^{3}(0)=g_{A}=F+D=1.26, \quad G_{1}^{8}(0)=(3 F-D) / \sqrt{3}=0.32 .
$$

The isosinglet charge $G_{1}^{0}(0)$ is again sensitive to the strange quark content and can e.g. be determined in polarized deep inelastic lepton scattering as discussed by Reya here [51] (see also ref.[36]). Alternatively, neutrino scattering off nucleons or nuclei might be used to get a handle on $G_{1}^{0}(0)$. This will be discussed below. At present, no experimental information is available about the $Q^{2}$-dependence of the singlet and octet axial ffs, so one either has to resort to some assumptions or models (see section 3.5). Clearly, the singlet axial charge and the singlet anomalous magnetic moment are the best objects to study the strange quark content of the nucleon using electroweak probes. The other obvious candidate is the so-called pion-nucleon $\sigma$-term which measures the strength of the strange matrix element $m_{s}<p|\bar{s} s| p>$. Combining $\pi N$ data, dispersion theory and constraints from chiral symmetry, Gasser et al. [52] have given the most precise determination of this quantity and its $t$-dependence. From this analysis one deduces that $m_{s}<p|\bar{s} s| p>\simeq 130 \mathrm{MeV}$. This is a sizeable though not dramatic strange quark effect. After defining the electroweak form factors, I will now discuss some experiments which allow to extract them and the related strange quark matrix elements. 


\subsection{Parity-Violating Electron Scattering}

Consider the scattering of polarized electrons on a nucleon or a nucleus. Due to the $\gamma-$ $Z^{0}$ interference, parity violation (pv) occurs and this allows to determine combinations of the electromagnetic and weak neutral ffs. The basic Feynman diagrams are simply the one-photon exchange proprotional to $F_{1,2}^{\gamma}$ and the $Z^{0}$-exchange proportional to $F_{1,2}^{Z}$ and $G_{1}$. To get an idea about the magnitude of this interference effect, let us perform some dimensional analysis. The elctromagnetic amplitude reads

$$
\mathcal{A}_{\mathrm{em}}=<f\left|J_{\mathrm{em}}^{\mu} \frac{1}{Q^{2}} j_{\mu, \mathrm{em}}\right| i>\sim \frac{\alpha}{Q^{2}},
$$

where $j_{\mu, \mathrm{em}}$ is the well-known em lepton current, $1 / Q^{2}$ the photon propagator and $J_{\mathrm{em}}^{\mu}$ the hadronic vector current parametrized by the em ffs. The parity-violating weak amplitude takes the form

$$
\mathcal{A}_{\mathrm{w}}^{\mathrm{pv}}=<f\left|V^{\mu} a_{\mu}+A^{\mu} v_{\mu}\right| i>\sim \frac{G_{F}}{\sqrt{2}}
$$

which is a product of the lepton $\left(v_{\mu}, a_{\mu}\right)$ and hadron $\left(V_{\mu}, A_{\mu}\right)$ vector and axial-vector currents, the latter containing the weak form factors. $G_{F}=1.16 \cdot 10^{-5} \mathrm{GeV}^{-2}$ is the Fermi constant. The total cross section can approximately be written as

$$
\sigma=\left|\mathcal{A}_{\mathrm{em}}+\mathcal{A}_{\mathrm{w}}^{\mathrm{pv}}\right|^{2} \simeq\left|\mathcal{A}_{\mathrm{em}}\right|^{2}+2\left|\mathcal{A}_{\mathrm{em}}^{*} \mathcal{A}_{\mathrm{w}}^{\mathrm{pv}}\right|
$$

The observable of interest is the left-right asymmetry $A$,

$$
A=\frac{\sigma_{R}-\sigma_{L}}{\sigma_{R}+\sigma_{L}} \simeq \frac{\left|\mathcal{A}_{\mathrm{em}}^{*} \mathcal{A}_{\mathrm{w}}^{\mathrm{pv}}\right|}{\left|\mathcal{A}_{\mathrm{em}}\right|^{2}} \sim \frac{G_{F} m^{2}}{4 \pi \alpha} \frac{Q^{2}}{m^{2}}=1.1 \cdot 10^{-4} \frac{Q^{2}}{\mathrm{GeV}^{2}} .
$$

For a typical four-momentum of $Q^{2}=0.1 \mathrm{GeV}^{2}$ in the non-perturbative regime we can expect an asymmetry of $10^{-5}$. This is a small number but within the reach of present day technology. Historically, the first measurement of this asymmetry using a deuterium target at SLAC was performed at $Q^{2}$ around $20 \mathrm{GeV}^{2}$ [53]. To be specific, consider now as the target the proton. An elementary calculation gives

$$
\begin{aligned}
A_{\mathrm{e} p} & =-\frac{G_{F} Q^{2}}{\sqrt{2} \pi \alpha} \frac{\left[\epsilon G_{E}^{\gamma} G_{E}^{Z}+\tau G_{M}^{\gamma} G_{M}^{Z}-\frac{1}{2}\left(1-4 s^{2}\right) \sqrt{1-\epsilon^{2}} \sqrt{\tau(1+\tau)} G_{M}^{\gamma} G_{1}\right]}{\epsilon\left(G_{E}^{\gamma}\right)^{2}+\tau\left(G_{M}^{\gamma}\right)^{2}} \\
& =-\frac{G_{F} Q^{2}}{\sqrt{2} \pi \alpha}\left[A_{E}+A_{M}+A_{A}\right]
\end{aligned}
$$

with $1 / \epsilon=\left[1+2(1+\tau) \tan ^{2}(\theta / 2)\right]=0 \ldots 1$ given by the scattering angle of the electron. In the second line, I have split the asymmetry into an electric, magnetic and axial piece. It is instructive to consider various kinematical regimes. At forward angles $(\theta \rightarrow 0, \epsilon \rightarrow 1)$, the electric part dominates at low $Q^{2}$ and the asymmetry is thus sensitive to the strange electric ff. At higher $Q^{2}$, due to the $\tau$ prefactor, the magnetic part takes over and one can access $G_{M}^{s}\left(Q^{2}\right)$. Notice that in forward direction the axial contribution is not only suppressed by $1-4 s^{2} \simeq 0.08$ but also by $\sqrt{1-\epsilon^{2}}$. In backward direction, matters are different since as $\theta \rightarrow 0, \epsilon$ also tends to zero. So at low $Q^{2}$ one can get $G_{M}^{s}\left(Q^{2}\right)$ with some contamination from the axial part. This 
is the kinematical regime where the SAMPLE experiment [54] hopes to determine the strange anomalous magnetic moment. This warrants a closer look. In a simplified analysis, we set $s^{2}=1 / 4, \theta=\pi$ and work at some low $Q^{2}$, say $0.2 \mathrm{GeV}^{2}$. Then, $A\left(Q^{2}\right)$ takes the simple form $[55]$

$$
A\left(Q^{2}\right) \simeq-\frac{G_{F} Q^{2}}{\sqrt{2} \pi \alpha} \frac{G_{M}^{Z}}{G_{M}^{\gamma}} \simeq-\frac{G_{F} Q^{2}}{4 \sqrt{2} \pi \alpha} \frac{\kappa_{p}-F_{2}^{0}(0)}{\kappa_{p}+1}=-\frac{G_{F} Q^{2}}{4 \sqrt{2} \pi \alpha} R
$$

assuming that the $Q^{2}$-dependence is similar for $G_{M}^{Z}$ and $G_{M}^{\gamma}$. The value of $R$ ranges form $R=1$ to $R=0.28$ for $F_{2}^{0}(0)=-1 \ldots 1$. For no strangeness contribution, we have $F_{2}^{0}(0)=-0.12$ and thus $R=0.64$. To give an idea about what one can expect, let me consider some models. The $S U(3)$ Skyrme model with a proper treatment of the symmetry breaking leads to $F_{2}^{0}(0)=-0.17 \ldots-0.25[56]$, the NJL model to $F_{2}^{0}(0)=-0.16 \ldots-0.26[57]$ and vector meson dominance supplemented with the $\phi$-meson (i.e. explicit strange quarks) to $F_{2}^{0}(0)=-0.43 \pm 0.09$ [58]. Similarly, one can also discuss the strange electric radius, i.e. the radius related to the operator $\bar{s} \gamma_{\mu} s$. A simple model based on a kaon cloud surrounding the nucleon leads to processes like $p \rightarrow \Lambda K^{+}, \Sigma^{0} K^{+}, \ldots \rightarrow p$ and lets us expect that $\left\langle r_{E}^{2}\right\rangle_{s}$ is positive since the positive charge sits in the cloud of the kaons. This is, however, a very crude estimate. In fact, most models tend to give a small and negative $\left\langle r_{E}^{2}\right\rangle_{s}$, like e.g. VMD plus the $\phi$-meson $-0.14 \pm 0.04 \mathrm{fm}^{2}[58]$ or the $S U(3)$ Skyrmion $-0.10 \pm 0.05 \mathrm{fm}^{2}$ [56]. These are fairly small numbers and it appears doubtful that the strange electric radius can be determined accurately from a proton target in the near future (assuming, of course, that its value is as small as indicated by the models). Here, nuclei might come in handy. For isoscalar, spin-0 nuclei such as ${ }^{4} \mathrm{He}$ or ${ }^{12} \mathrm{C}$, one finds for the asymmetry $[59]$

$$
A=\frac{G_{F} Q^{2}}{4 \sqrt{2} \pi \alpha}\left[s^{2}+\frac{G_{E}^{s}}{2\left(G_{E}^{p}+G_{E}^{n}\right)}\right],
$$

which at very low $Q^{2}$ allows to extract the weak mixing angle and at somewhat higher momenta the strange electric ff. Of course, one has to take into account nuclear structure issues like pv level mixing and so on. A lucid discussion of these topics can be found in refs. $[59,60]$. Clearly, pv electron scattering is an interesting field and many more aspects of it not covered here can e.g. be found in the proceedings of the CALTECH workshop [61].

\subsection{Neutrino and Antineutrino Scattering off Nucleons and Nuclei}

Neutrino scattering off nucleons or nuclei offers another possibility of exploring the strange quark content of the nucleon. The neutrino couples via the $Z^{0}$ to the quarks and thus probes isosinglet, triplet and octet components. Let us first consider the differential cross section for elastic neutrino/antineutrino-proton scattering,

$$
\frac{d \sigma}{d Q^{2}}=\frac{G_{F}^{2} m^{2}}{8 \pi E_{\nu}^{2}}\left[A \pm B W+C W^{2}\right]
$$

with $E_{\nu}$ the neutrino energy, $W=\left(4 E_{\nu} m-Q^{2}\right) / m^{2}$ and the ' $\pm^{\prime}$ refers to the case of neutrinos (antineutrinos). The functions $A, B$ and $C$ depend on the ffs $G_{1}$ and $F_{1,2}^{Z}$ (I omit the superscript ' $Z$ ' on $F_{1}$ and $F_{2}$ ), 


$$
\begin{aligned}
& A=4 \tau\left[G_{1}^{2}(1+\tau)-4(1-\tau)\left(F_{1}^{2}-\tau F_{2}^{2}\right)+16 \tau F_{1} F_{2}\right], \\
& B=-8 \tau G_{1}\left(F_{1}+F_{2}\right) \\
& C=G_{1}^{2} / 4+F_{1}^{2}+\tau F_{2}^{2} .
\end{aligned}
$$

To demonstrate this in more detail, let me simplify the analysis by assuming a $Q^{2}$ value of about $0.5 \mathrm{GeV}^{2}$ and retaining only the terms linear in the strange ffs. This allows to recast (3.24) in the form [62]

$$
\frac{d \sigma}{d Q^{2}}=\sigma_{\text {non-strange }}\left[1-0.72 F_{1}^{s}\left(Q^{2}\right)-0.78 F_{2}^{s}\left(Q^{2}\right)-1.23 G_{1}^{s}\left(Q^{2}\right)\right]
$$

Obviously, to extract the strange matrix element $<p\left|\bar{s} \gamma_{\mu} \gamma_{5} s\right| p>=G_{1}^{s}(0)$, one has to know something about $F_{1,2}^{s}\left(Q^{2}\right)$ or make some assumptions. Ahrens et al. [63] and Kaplan and Manohar [50] have shown that setting $F_{1}^{s}=F_{2}^{s}=0$ and assuming that all three axial ffs have the same dipole mass $\left(M_{A}=1.032 \mathrm{GeV}\right)$, the extracted value of $G_{1}^{s}(0)$ is in agreement with the one obtained from polarized deep inelastic $\mu p$ scattering [36]. However, as already stressed in ref.[63], this result is very sensitive to the actual value of the diple mass $M_{A}$. This issue was further addressed by Bernard et al. [64]. They used the topological chiral soliton model of the nucleon to calculate the isosinglet axial $\mathrm{ff}$ since in the framework of this model the known triplet $\mathrm{ff}$ is well described. It was found that the cut-off mass in the singlet channel is actually 20 per cent larger than the one for the triplet and octet ffs. Redoing the analysis with these ffs, one finds that the value of $G_{1}^{s}(0)$ is reduced by a factor of three. A similar analysis has recently been performed by Garvey et al.[65]. In conclusion, the often claimed agreement concerning the value of $G_{1}^{s}(0)$ from the neutrino and EMC data can only be considered accidental. To avoid the problems of extrapolating from the typical $Q^{2}$ of 0.5 to $1 \mathrm{GeV}^{2}$ in elastic $\nu p$ or $\bar{\nu} p$ scattering, it was recently proposed that (anti)neutrino induced quasi-free nucleon knock-out off nuclei might give a better handle on the strange matrix elements [66]. In the quasi-free region, one has small momentum transfers and typical neutrino energies of $0.2 \mathrm{GeV}$. The ratio of the proton to the neutron yield depends sensitively on $G_{1}^{s}$ and $F_{2}^{s}$,

$$
R=Y_{p} / Y_{n}=F\left(G_{1}^{s}, F_{2}^{s}\right) .
$$

For example, if $F_{2}^{s}(0)=-0.22, R_{\bar{\nu}}$ varies between 0.85 and 1.9 for $G_{1}^{s}(0)$ between 0 and -0.2. For further details, see ref.[66]. Of course, it is mandatory to understand well the nuclear structure issues, in particular one assumes that the knock-out is involving only one nucleon. How good these assumptions are is still under debate. What is important is to realize that neutrino and pv electron scattering processes nicely complement each other and that many different experiments have to be performed to ultimately pin down the strength of the strange matrix elements in the proton. 


\subsection{Down and Dirty: QED, QCD and Heavy Quark Corrections}

Up to now, I have entirely worked at tree level. At low energies, radiative corrections and effects from heavy quarks are expected to be small. However, in certain cases the tree level couplings are suppressed or vanish (as it is the case with the isocalar axial current) or one is trying to extract small numbers. Therefore, it is mandatory to investigate the effects of QED and QCD corrections on the processes considered before. Since the neutrino has no direct QED and QCD couplings, let me start with the discussion of corrections to neutrino-hadron (quark) scattering. To be specific, consider the induced isoscalar coupling. At tree level it vanishes, but that does not mean that it is zero alltogether. The basic Feynman diagram which induces such a coupling is the famous triangle diagram. On one end, the axial part $\left(\gamma_{\mu} \gamma_{5}\right)$ of the neutrino current couples and the other two corners are attached via gluon exchanges to the external light $(\mathrm{u}, \mathrm{d}, \mathrm{s})$ quark. In the loop, quarks of all flavors run around. A simple one-gluon exchange is forbidden by color neutrality. This is the basic diagram to lowest order in the strong interactions. The contribution of such a type of diagram has to be finite since the sum over all quark flavors leads to anomaly cancellation,

$$
\sum_{i} T_{3}^{i}=0
$$

where $T_{3}$ denotes the weak isospin. However, we are interested at scales much below the intermediate vector boson and heavy quark masses. In this energy regime, the triangle diagram leads to an induced isoscalar current which is not suppressed by inverse powers of the heavy quark masses as naive decoupling would suggest. Indeed, a straightforward perturbative calculation of this two-loop diagram yields [67]

$$
A_{\mu}^{Z}=\frac{1}{2}\left(A_{u}-A_{d}\right)+\left(A_{u}+A_{d}\right) \frac{1}{4}\left(\frac{g^{2}}{4 \pi}\right)^{2} \ln \frac{\Pi_{i} m_{i+}^{2}}{\Pi_{i} m_{i-}^{2}},
$$

where $m_{i \pm}$ refers to the masses of the quarks with weak isospin $\pm 1 / 2$, i.e. with charges $2 / 3$ and $-1 / 3$, respectively, and $g$ is the strong coupling constant. Notice that the induced isoscalar current $\left(A_{u}+A_{d}\right)$ depends logarithmically on the quark masses and not on inverse powers of them (as advertised). Collins, Wilczek and Zee [67] have shown how to do this in a more systematic fashion. Their method is based on integrating out quark doublets in succession. Denote by $H=A_{t}-A_{b}$ the axial current of the heaviest doublet. The idea is to recast $H$ in the form

$$
H=\sum_{i} A_{i} \bar{L}_{i}+\sum_{i} B_{i} \bar{H}_{i}
$$

where the light $\left(\bar{L}_{i}\right)$ and the heavy $\left(\bar{H}_{i}\right)$ particle operators exhibit decoupling. This means that the matrix elements of the $\bar{L}_{i}$ see the heavy quarks only via power law corrections. Repeating this exercise for $\tilde{H}=A_{c}-A_{s}$, the induced isoscalar current reads

$$
\Delta A_{\mu}^{Z}=\left(A_{u}+A_{d}\right) \frac{1}{4} \frac{\alpha_{s}\left(m_{s}\right)}{\pi} \frac{\alpha_{c}\left(m_{c}\right)}{\pi} \ln \frac{m_{c}^{2}}{m_{s}^{2}} \simeq 0.05\left(A_{u}+A_{d}\right),
$$

where I have neglected the much smaller contribution from the $(t, b)$ doublet. Of course, this approach is too bold since at the scale of the strange quark mass $\lambda=$ 
$m_{s} \simeq 175 \mathrm{MeV}$, this perturbative treatment can not be justified any more. Therefore, Kaplan and Manohar [50] have generalized the method of ref.[67] to integrate out the various quarks separately. Their argument is based on the assumption $M_{Z}>m_{t}$. This simplifies the analysis but is not mandatory. In this case, integrating out the $Z^{0}$ one has an effective Lagrangian for neutrino-quark scattering,

$$
\mathcal{L}_{\text {eff }}=-\frac{G_{F}}{\sqrt{2}} \sum_{u, d, s, c, b, t} \bar{\nu} \gamma^{\mu}\left(1-\gamma_{5}\right) \nu\left[\bar{q}\left(T_{3}-2 s^{2} Q\right) \gamma_{\mu} q-\bar{q} T_{3} \gamma_{\mu} \gamma_{5} q\right]
$$

In principle, one should now calculate corrections to this effective Lagrangian. However, due to the absence of direct QED or QCD couplings of the neutrino, one can rewrite these corrections as corrections to the hadronic current. The procedure goes as follows. One uses the renormalization group to evolve (3.30) down to the scale of the top mass. At that energy, one integrates out the $t$ quark. This induces two types of corrections, one class scaling like $g^{2}\left(m_{t}\right) / m_{t}^{2}$ (i.e. exhibiting decoupling). Since the $\mathrm{t}$ is removed from the theory, the $Z^{0}$ axial current has an anomaly because (3.26) is not fulfilled any more. This leads to a multiplicative renormalization of the singlet axial current, which is the most important effect as we will see. One then proceeds by scaling down to $\lambda=m_{b}$, integrating out the $\mathrm{b}$ and so on until one reaches the scale $\lambda=1 \mathrm{GeV}$ (which is a typical energy in the elastic $\nu p$ scattering process and large enough to justify the perturbative treatment). Putting the important pieces from the multiplicative renormalizations together, the relation between the axial current at $\lambda=M_{Z}$ and at $\lambda=1 \mathrm{GeV}$ reads [50]

$$
\begin{aligned}
A_{\mu}^{Z}(\lambda=1 \mathrm{GeV}) & =A_{\mu}^{Z}\left(\lambda=M_{Z}\right)+\frac{1}{2}\left(A_{u}+A_{d}\right) \Delta_{A} \\
\Delta_{A} & =\Lambda_{\mu c}\left[\frac{1}{2}+\frac{3}{10} \Lambda_{c b}\left(\Lambda_{b t}-1\right)\right] \\
\log \Lambda\left(m_{i}, m_{j}, N_{f}\right) & =N_{f} \frac{\alpha_{s}\left(m_{i}\right)}{\pi} \frac{\alpha_{s}\left(m_{j}\right)}{\pi} \log \frac{m_{i}^{2}}{m_{j}^{2}},
\end{aligned}
$$

which leads to $\Delta A=0.02$ [50]. Obviously one recovers the result (3.29) if one were to integrate out the s quark too. Notice that the finite pieces due to the renormalization group evolution are negligible and that there is also an induced vector current with $\Delta_{V}<10^{-4}$. The main effect of the photons comes from the penguin diagram leading to a running of the weak mixing angle [68]

$$
s^{2}\left(\lambda^{2}\right)=s^{2}\left(M_{W}^{2}\right)+\sum_{j} \frac{\alpha}{3 \pi} \ln \left(\frac{M_{W}^{2}}{\lambda^{2}}\right) Q_{j}\left(T_{3 j}-2 s^{2} Q_{j}\right),
$$

where the index $j$ extends over the quark flavours which are integrated out. In addition, there are pure next-to-leading order electroweak effects. These have been considered in ref.[69]. The radiatively induced isoscalar axial current has a very small coefficient,

$$
-\frac{3 \alpha}{16 \pi s^{2}}\left[1+\frac{1}{2 c^{2}}\left(1-2 s^{2}+\frac{20}{9} s^{4}\right)\right]=-0.003,
$$

which is negligible compared to the heavy quark effects. Therefore, if one restrcits oneself to scales above $\lambda \simeq 1 \mathrm{GeV}$, the QED and QCD corrections to neutrino-hadron 
scattering are known and under control. Matters become more messy if one attempts to go to lower energies and also if one considers electron-hadron scattering as I will do now. In this case, we have in addition the direct photon exchange which gives rise to further complications. What we are after can be summarized as follows. Denote by $C$ the tree level value of any coupling between the lepton and the hadron (quark) currents. Due to the radiative corrections, these couplings are modified

$$
C=C[1+R],
$$

so that $R$ gives the ratio of the corresponding value including radiative corrections to the tree level one (in case that $C$ happens to vanish at tree level, one has to choose another coupling as the reference point). Naively, one expects the size of these corrections to be small, the typical scale being

$$
G_{F} m^{2} \frac{\alpha}{4 \pi} \leq 10^{-8}
$$

This rather small number is sometimes considerably enhanced and, furthermore, the radiative corrections can in some circumstances compete with the effects induced by the strange quarks. Holstein and Musolf [70] have given the most detailed evaluation of these effects. Clearly, the results of their calculations should be considered indicative since considerable uncertainties are involved as will be discussed below. A very basic and introductory presentation concerning the calculation of radiative corrections for the pv processes under consideration has been given by Musolf [71] which the reader not familiar with the concepts of renormalization in the standard model certainly will appreciate. In essence, there are two classes of Feynman diagrams contributing at one loop order. The first class involves only one quark in the nucleon. Typical representatives are vertex or vector boson propagator corrections. More complicated are the diagrams involving two quarks, such as the $\gamma Z^{0}$ box or exchange current type diagrams where a massive vector bosons is exchanged between two quarks while the photon couples to one of them (many pictorials are displayed in ref.[71]). Let me first consider the one quark type diagrams. These depend on the not yet known masses of the $t$ quark and the Higgs boson and, if there is physics beyond the standard model, implicetely on the parameters of this new physics. This latter dependence is parametrized in terms of $\mathrm{S}, \mathrm{T}$ and $\mathrm{U}$ or $\epsilon_{1}, \epsilon_{2}$ and $\epsilon_{3}$ (as discussed by Altarelli here [49]). There are two effects which can conspire to give much larger values for these loop corrections than the estimate (3.35) would suggest. These are large logarithms of the light fermion to the vector boson mass ratios and the suppression of tree level couplings. A particularly illustrative example is the scattering process $\nu_{e} \mu \rightarrow$ $\nu_{e} \mu$. While the tree level $Z^{0}-\mu$ coupling is suppressed, the $W$-vertex correction to the $Z^{0}-\nu_{e}$ coupling induces a $\log \left(m_{e}^{2} / M_{W}^{2}\right)$, with $m_{e}$ the electron mass. The corresponding ratio follows to be [70]

$$
R\left(\nu_{e} \mu \rightarrow \nu_{e} \mu\right)=\frac{\alpha}{4 \pi} \frac{8}{3} \frac{\ln \left(m_{e}^{2} / M_{W}^{2}\right)}{4 s^{2}-1} \simeq 0.5 .
$$

This calculation is clean in that it involves only leptons. For the case of electronhadron scattering, the quarks are bound within a nucleon of a size of about $1 \mathrm{fm}$. Using the uncertainty principle, this allows one to get an idea about the typical quark momenta. However, this is a very crude estimate and induces some uncertainty (for 
details, see ref.[70]). Furthermore, it is not obvious which quark mass values one should insert. When one considers quark loop corrections to the vector boson propagators, one obviously deals with the current quarks. In contrast, in the various box diagrams it is more justified to use the so-called constituent masses $(\sim 330 \mathrm{MeV}$ for the $\mathrm{u}$ and d quarks) to account for the binding effects. These subtleties are also discussed in refs.[70,71]. For processes of the type $V(e) \times A(N)$ (which means that the vector current of the electron couples to the nucleonic axial current), one finds corrections $R^{p}=-0.65 \ldots-0.28$ and $R^{n}=-0.56 \ldots-0.06$ for the proton and the neutron, in order. These are clearly larger than the dimensional argument (3.35) suggests. Furthermore, we also have to consider the many quark diagrams. Here, the situation is less transparent, i.e. it is much more difficult to deal with this class. To proceed, one can resort to some kind of "hadronic duality" [70,71,72]. This means that one considers a meson cloud picture instead of the more complex quark diagrams. As examples, the excitation of quark-antiquark pairs with the quantum numbers of a pion probed by the photon and with a successive vector boson exchange translates to the photon coupling to a pion in flight, with one pion-nucleon coupling parityconserving (strong) and the other pv (weak). Similarly, other diagrams translate into a $\gamma \rho^{0}$ conversion followed by a pv $\rho N$ coupling. Such diagrams contribute to the nucleon axial current via [70,72]

$$
\begin{aligned}
& \delta A_{N}^{\pi}=\frac{g_{\pi N} h_{\pi N}}{m^{2}}\left[\frac{\pi}{6} \frac{m}{M_{\pi}}+\ln \left(\frac{M_{\pi}}{m}\right)+\ldots\right], \\
& \delta A_{N}^{\rho}=\frac{h_{\rho N}}{g_{\rho N}} \frac{1}{m_{\rho}^{2}} .
\end{aligned}
$$

The strong coupling constants $\left(g_{\pi N}, g_{\rho N}\right)$ are fairly well known. Matters are different for the parity-violating couplings $\left(h_{\pi N}, h_{\rho N}\right)$. Their calculation has been pioneered by Donoghue et al.[73], a recent update including additional experimental constraints and chiral soliton model calculations can be found in refs.[74.75]. The pion cloud contribution diverges in the chiral limit which is no surprise in the light of the discussion presented in section 2. Ultimately, CHPT methods might shed some light on these long-distance contributions. In ref.[70], the estimates for processes of the type $V(e) \times A(N)$ range from $-0.07 \ldots+0.37$ for $R^{p}$ and $-0.07 \ldots+0.24$ for $R^{n}$. Combining these numbers with the ones from the one quark diagrams, one sees that there are potentially large corrections. To make all this more transparent, let us consider again the determination of the strange anomalous magnetic moment from pv electron-proton scattering. In backward direction and at small $Q^{2}$ we have [76]

$$
A_{e p} \simeq-\frac{G_{F} Q^{2}}{4 \sqrt{2} \pi \alpha}\left\{\frac{\kappa_{p}}{\mu_{p}}\left[1+R_{\text {strange }}^{M}+R_{\text {rad }}^{M}\right]-\frac{2 m\left(E^{\prime}+E\right)}{Q^{2}} \frac{1-4 s^{2}}{\mu_{p}} g_{A}\left(1+R_{p}^{A}\right)\right\},
$$

where $R_{\mathrm{strange}, \text { rad }}^{M}$ denotes the strangeness and radiative corrections to the magnetic moment and $R_{p}^{A}$ the one to the proton axial current. Two important observations are in order. First, the radiative corrections to the magnetic asymmetry are comparable to the strange quark induced ones,

$$
\frac{R_{\mathrm{rad}}^{M}}{R_{\mathrm{strange}}^{M}} \simeq \frac{0.1}{\mu_{s}}
$$


Second, there is a large correction to the background of the axial asymmetry which makes up roughly 30 percent of the signal for the SAMPLE kinematics. These two effects severely constrain the accuracy for determining the strange anomalous magnetic moment. To overcome these problems, it is therefore mandatory to perform many complementary experiments like e.g. backward-angle e $d$ scattering, pv quasielastic scattering and so on. For a nice overview about these topics, I refer to ref.[76]. Finally, let me stress again that more theoretical effort is needed to further tighten the limits on these radiative corrections so that an unambigous interpretation of the experiments will be possible.

\section{Summary and Outlook}

The standard model of the strong and electroweak interactions is an embarrasingly successful theory. It is least understood at its extreme energy limits. First, at very high energies, it is not known what exactly triggers the spontaneoaus symmetry breaking of $S U(2)_{L} \times U(1)_{Y} \rightarrow U(1)_{\mathrm{em}}$ at a scale of $\langle\phi\rangle=250 \mathrm{GeV}$. While the standard Higgs boson does the job, one is left with just too many free parameters to feel confident with such a scenario. At present, the physics beyond the standard model hides itself quite effectively as discussed by Altarelli at this school [49]. Future high-energy colliders like the LHC or the SSC will hopefully shed light on the electroweak symmetry breaking sector. May be less spectacular, but as challenging is the problem of hadron structure in the non-perturbative regime. Chiral perturbation theory is a method which allows to work out systematically the consequences of the spontaneous chiral symmetry breaking in QCD. It is based on a simultaneous expansion in the (small) external momenta and (light) quark masses $(\mathrm{u}, \mathrm{d}, \mathrm{s})$ and enjoys considerable success in the meson sector. In the first part of these lectures, I have mostly been concerned with the problems surrounding the inclusion of matter fields in the chiral expansion. While this is technically a straightforward procedure based on chiral counting rules, it is conceptually less transparent than in the meson sector due to the appearance of the nucleon mass term. This is a scale of the order of the chiral symmetry breaking scale also it is not related to the symmetry violation and, furthermore, it does not vanish in the chiral limit. I have concentrated here on a few selected processes in the two-flavor sector like nucleon Compton scattering or pion photo- and electroproduction. Since the $\mathrm{u}$ and $\mathrm{d}$ quarks are really light, the corresponding expansion parameters $M_{\pi} / 4 \pi F_{\pi}$ and $E_{\text {pion }} / F_{\pi}$ are small and one has a better change of a converging chiral expansion. However, as we have seen in the discussion of the LET (2.14), the appearance of the nucleon mass renders these dimensional arguments rather dangerous. It appears at this point that in the heavy mass formulation of baryon CHPT such terms should not show up since the baryon propagator does not include the nucleon mass. However, in that case the large new scale is hidden by means of the Goldberger-Treiman relation in large prefactors accompanying the axial-vector coupling strength $g_{A}$. All calculations performed so far have been carried out in the one-loop approximation indicating that it is mandatory to include at least the terms of order $q^{4}$ (which are beyond next-toleading order). This argument is further strengthened when one considers the three-

flavor sector. Here, one very often finds large kaon and eta loop corrections which make one feel uneasy about the validity of the chiral expansion [77]. The suggestion 
that these large loop effects are largely cancelled by contributions from the spin- $3 / 2$ decuplet in the intermediate states $[16,18]$ is appealing but has not yet been put on a firm basis, which means that one has to perform a full order $q^{4}$ (or higher) calculation. There are, however, on-going activities in this area and further progress can be expected soon.

The second topic of these lectures concerned the quark structure of the nucleon at low energies. I have discussed how particular combinations of the electromagnetic and weak form factors, which parametrize the structure of the nucleon and its flavor decomposition, allow to extract interesting matrix elements like the strange anomalous magnetic moment, $F_{2}^{s}(0)$, or the strange axial nucleon charge, $G_{1}^{s}(0)$. Parity-violating electron scattering ( $\gamma Z^{0}$-interference) and (anti)neutrino scattering off nucleons or nuclei are the tools to determine these and other matrix elements like e.g. the extension of the nucleon as given by the strange electric radius. However, and that was a major topic here, since in most cases one tries to extract small numbers, one also has to account for radiative and heavy quark corrections. While these are nominally small, they often tend to be enhanced by large factors way above the naive dimensional estimates. In the most extreme case (cf. the isoscalar axial coupling), the tree level couplings with which one works at leading order are vanishing. In neutrinohadron scattering, if one does not go below a typical scale of say $1 \mathrm{GeV}$, these various corrections are under control. As a particular example, I have discussed the induced isoscalar axial coupling, which is not only empirically interesting but also shows how under certain circumstances effective field theory methods can and should be used to caluclate QED, QCD and heavy quark corrections [50]. When it comes to electronhadron scattering, the situation is much less satisfactory. First, as I discussed, in many experiments one wants to work at much smaller energy scales and therefore has to account for the effects of quark confinement. Second, the direct photon coupling to the hadrons (quarks) gives rise too much more diagrams than it is the case in neutrino scattering. The most systematic analysis of radiative corrections to parityviolating electron-hadron scattering [70] translates the Feynman diagrams involving more than one quark in the nucleon into "hadronic ones", i.e. making use of the meson cloud picture. This induces uncertainties which are of the order of the calculated effects themselves. Another source of uncertainty is the momentum distribution of the bound quarks which one can only account for in an approximate fashion. Obviously, the theorists are called for providing better calculations of this type so that the experimenters have a better change to extract the various strange matrix elements. Further complications arise when one considers the scattering off nuclei [78]. As I pointed out, many complimentary experiments which are sensitive to different combinations of the form factors and have different next-to-leading order corrections have to and will be performed at MAMI, CEBAF, ... [79]. These experiments are eagerly awaited. To come back to the first part of the lectures, once we will have learned how to treat the three-flavor sector of baryon CHPT, one will also be able to discuss the strange matrix elements and confront the theoretical predictions with the data. 


\section{Acknowledgements}

I would like to thank the organizers, in particular Professor W. Plessas, for their invitation and kind hospitality extended to me. This work was supported in part

by the Deutsche Forschungsgemeinschaft through a Heisenberg fellowship and the Schweizerischer Nationalfonds.

\section{References}

[1] J. Goldstone, Nuovo Cim. 19 (1961) 154

[2] H. Leutwyler, in "Recent Aspects of Quantum Fields", eds. H. Mitter and M. Gausterer, Springer Verlag, Berlin, 1991

[3] Ulf-G. Meißner, Recent Developments in Chiral Perturbation Theory, preprint BUTP93/01 (1993)

[4] S. Weinberg, Phys. Rev. Lett. 17 (1966) 616

[5] H. Lehmann, Phys. Lett. B41 (1972) 529; Acta Phys. Austriaca Suppl. 11 (1973) 139

[6] S. Weinberg, Physica 96A (1979) 327

[7] J. Gasser and H. Leutwyler, Ann. Phys. (N.Y.) 158 (1984) 142

[8] G. Ecker, J. Gasser, A. Pich and E. de Rafael, Nucl. Phys. B321 (1989) 311; J. F. Donoghue, C. Ramirez and G. Valencia, Phys. Rev. D39 (1989) 1947

[9] J. Gasser and Ulf-G. Meißner, Phys. Lett. B258 (1991) 219

[10] J. Gasser and Ulf-G. Meißner, Nucl. Phys. B357 (1991) 90

[11] S. Coleman, J. Wess and B. Zumino, Phys. Rev. 177 (1969) 2239; C. G. Callan, S. Coleman, J. Wess and B. Zumino, Phys. Rev. 177 (1969) 2247

[12] A. Krause, Helv. Phys. Acta 63 (1990) 3

[13] J. Gasser, M.E. Sainio and A. Švarc, Nucl. Phys. B307 (1988) 779

[14] E. Jenkins and A.V. Manohar, Phys. Lett. B255 (1991) 558

[15] S. Weinberg, Nucl. Phys. B363 (1991) 3

[16] E. Jenkins and A.V. Manohar, in "Effective Field Theories of the Standard Model", ed. Ulf-G. Meißner, World Scientific, Singapore, 1992

[17] Ulf-G. Meißner, Int. J. Mod. Phys. E1 (1992) 561

[18] E. Jenkins and A.V. Manohar, Phys. Lett. B259 (1991) 353

[19] V. Bernard, N. Kaiser and Ulf-G. Meißner, in preparation

[20] N.M. Kroll and M.A. Ruderman, Phys. Rev. 93 (1954) 233

[21] V. Bernard, J. Gasser, N. Kaiser and Ulf-G. Meißner, Phys. Lett. B268 (1991) 291

[22] V. Bernard, N. Kaiser and Ulf-G. Meißner, Nucl. Phys. B383 (1992) 442

[23] A. M. Bernstein and B.R. Holstein, Comments Nucl. Part. Phys. 20 (1991) 197

[24] D. Drechsel and L. Tiator, J. Phys. G: Nucl. Part. Phys. 18 (1992) 449

[25] V. Bernard, N. Kaiser and Ulf-G. Meißner, Phys. Lett. B282 (1992) 448

[26] J. H. Koch, S. Scherer and J. L. Friar, to be published, and S. Scherer and J. H. Koch, Nucl. Phys. A534 (1991) 461

[27] T. P. Welch et al., Phys. Rev. Lett. 69 (1992) 2761

[28] V. Bernard, N. Kaiser, T.-S. H. Lee and Ulf-G. Meißner, Phys. Rev. Lett. 70 (1993) 387

[29] Y. Nambu and D. Lurié, Phys. Rev. 125 (1962) 1429; Y. Nambu and E. Shrauner, Phys. Rev. 128 (1962) 862

[30] V. Bernard, N. Kaiser and Ulf-G. Meißner, Phys. Rev. Lett. 69 (1992) 1877 
[31] F.J. Federspiel et al., Phys. Rev. Lett. 67 (1991) 1511

[32] F.E. Low, Phys. Rev. 96 (1954) 1428; M. Gell-Mann and M.L. Goldberger, Phys. Rev. 96 (1954) 1433

[33] V. Bernard, N. Kaiser, J. Kambor and Ulf-G. Meißner, Nucl. Phys. B388 (1992) 315

[34] R.L. Workman and R.A. Arndt, Phys. Rev. D45 (1992) 1789

[35] S.D. Drell and A.C. Hearn, Phys. Rev. Lett. 16 (1966) 908; S.B. Gerasimov, Sov. J. Nucl. Phys. 2 (1966) 430.

[36] J. Ashman et al., Phys. Lett. B206 (1988) 364.

[37] V. Bernard, N. Kaiser and Ulf-G. Meißner, Bern University preprint BUTP-92/51, 1992

[38] V. Burkert and Z. Li, Phys. Rev. D47 (1993) 46

[39] G. Höhler, in Landolt-Börnstein, vol.9 b2, ed. H. Schopper, Springer, Berlin, 1983

[40] H. Arenhövel, these proceedings; B. Schoch, these proceedings

[41] R.E. Taylor, these proceedings

[42] S. Platchkov et al., Nucl. Phys. A508 (1990) 343

[43] M. Gari and W. Krümpelmann, Phys. Lett. B274 (1992) 159; A. Lung et al., Phys. Rev. Lett. 70 (1993) 718

[44] S.J. Brodsky and G.P. Lepage, Phys. Rev. D22 (1980) 2157 and references therein

[45] Ulf-G. Meißner, Phys. Rep. 161 (1988) 213

[46] D. Beck, Phys. Rev. D39 (1989) 3248

[47] R. Cahn and F. Gilman, Phys. Rev. D17 (1978) 1313

[48] D. Beck, University of Illinois preprint NPL-91-015, 1991

[49] G. Altarelli, these proceedings

[50] D. Kaplan and A.V. Manohar, Nucl. Phys. B310 (1988) 527

[51] E. Reya, these proceedings

[52] J. Gasser, H. Leutwyler and M.E. Sainio, Phys. Lett. 253B (1991) 252, 260

[53] C.Y. Prescott et al., Phys. Lett. B77 (1978) 1347; Phys. Lett. B84 (1979) 524

[54] BATES experiment 89-06, see e.g. D. Beck in ref.[61]

[55] R.D. McKeown, Phys. Lett. B219 (1989) 40

[56] N.W. Park and H. Weigel, Nucl. Phys. A541 (1992) 453

[57] V. Bernard and Ulf-G. Meißner, Phys. Lett. B216 (1989) 392; B223 (1989) 439

[58] R.L. Jaffe, Phys. Lett. B229 (1989) 275

[59] M.J. Musolf and T.W. Donnelly, Nucl. Phys. A546 (1992) 509

[60] T.W. Donnelly et al., Nucl. Phys. A541 (1992) 525

[61] "Parity Violation in Electron Scattering", eds. E. Beise and R. McKeown, World Scientific Publ., Singapore, 1990

[62] E. Beise and R. McKeown, Comm. Nucl. Part. Phys. 20 (1991) 105

[63] L.A. Ahrens et al., Phys. Rev. D35 (1987) 785

[64] V. Bernard, N. Kaiser and Ulf-G. Meißner, Phys. Lett. B237 (1990) 545

[65] G.T. Garvey, W.C. Louis and D.H. White, Los Alamos preprint LA-UR-93-0037, 1992

[66] G.T. Garvey et al., Phys. Lett. B289 (1992) 249

[67] J. Collins, F. Wilczek and A. Zee, Phys. Rev. D18 (1978) 242

[68] H. Georgi, "Weak Interactions and Modern Particle Physics", Benjamin/Cummings, Reading, MA, 1984

[69] R.N. Mohapatra and G. Senjanovic, Phys. Rev. D19 (1979) 2165

[70] M.J. Musolf and B.R. Holstein, Phys. Lett. B242 (1990) 461

[71] M.J. Musolf in ref.[61]

[72] W.C.Haxton, E.M.Henley and M.J. Musolf, Phys. Rev. Lett. 63 (1989) 949

[73] B. Desplanques, J.F. Donoghue and B.R. Holstein, Ann. Phys. 124 (1980) 449

[74] N. Kaiser and Ulf-G. Meißner, Nucl. Phys. A499 (1989) 699 
[75] Ulf-G. Meißner, Mod. Phys. Lett. A5 (1990) 1703

[76] M.J. Musolf, MIT preprint CTP 2120, 1992

[77] J. Bijnens, H. Sonoda and M.B. Wise, Nucl.Phys. B261 (1985) 185

[78] C.J. Horowitz and J. Piekarewicz, Florida State University preprint, FSU-SCRI-9318, 1993

[79] Some examples are MAMI-A4, CEBAF 91-004, CEBAF 91-010 and CEBAF 91-017

This article was processed by the author using the $\mathrm{T}_{\mathrm{E}} \mathrm{X}$ macro package from Springer-Verlag. 